REVIEW ARTICLE

https://doi.org/10.1038/s41467-019-11707-7

\title{
Moving beyond the glial scar for spinal cord repair
}

\author{
Elizabeth J. Bradbury $\mathbb{1}^{1}$ \& Emily R. Burnside (i) ${ }^{1}$
}

\begin{abstract}
Traumatic spinal cord injury results in severe and irreversible loss of function. The injury triggers a complex cascade of inflammatory and pathological processes, culminating in formation of a scar. While traditionally referred to as a glial scar, the spinal injury scar in fact comprises multiple cellular and extracellular components. This multidimensional nature should be considered when aiming to understand the role of scarring in limiting tissue repair and recovery. In this Review we discuss recent advances in understanding the composition and phenotypic characteristics of the spinal injury scar, the oversimplification of defining the scar in binary terms as good or bad, and the development of therapeutic approaches to target scar components to enable improved functional outcome after spinal cord injury.
\end{abstract}

$\mathrm{t}$ is estimated that more than 27 million people worldwide are living with long-term disability following a spinal cord injury ${ }^{1}, 90 \%$ of which result from trauma and $10 \%$ as a secondary consequence of disease. Following traumatic spinal cord injury, death of spinal neurons at the injury level leads to paralysis of denervated musculature and the disruption of long spinal tracts leads to loss of sensation and motor control-injured descending axonal projections can no longer innervate motor neuron pools below the injury level, and injured ascending axonal projections can no longer provide appropriate transmission of sensory information to the brain. This results in the dysregulation of multiple organ systems throughout the body and a devastating loss of function ${ }^{2}$. Despite recent progress in developing experimental therapeutics aimed at enhancing tissue repair and neuroplasticity, there are still no effective pathology-modifying or regenerative treatments available to spinal injured individuals ${ }^{3,4}$. Following diagnosis and acute medical interventions to stabilize clinical status, the outcome is largely determined by the management of resultant symptoms, and rehabilitation to maximize residual neural function ${ }^{2}$.

The lack of repair following spinal cord injury is due to both cell intrinsic factors and the extrinsic injury environment. Neurons of the adult mammalian central nervous system (CNS) have low intrinsic regenerative ability due to a lack of growth driving signals, and suboptimal availability or arrangement of subcellular machinery to enable growth cone reformation and axonal elongation ${ }^{5}$. Experimental efforts to unlock regeneration potential at the level of the cell body of the neuron have focussed on growth signalling pathways, individual regenerationassociated genes, and transcriptional and epigenetic networks ${ }^{6-9}$. Regenerative strategies have also aimed to increase synthesis and transport of materials required for growth, and to modulate axonal cytoskeletal dynamics to promote elongation or branching $6,7,10-12$.

The injury microenvironment also plays a key role in limiting functional repair after spinal cord injury, an important component of which is the formation of a scar. As a healing response, the scar acts to spatially contain and isolate damage. However, reactive injury processes fail to restore spinal tissue architecture and composition, pathology continues to propagate and the tissue within and around the scar remains dysfunctional. Moreover, within the scar there are

\footnotetext{
${ }^{1}$ King's College London, Regeneration Group, The Wolfson Centre for Age-Related Diseases, Institute of Psychiatry, Psychology \& Neuroscience (loPPN), Guy's Campus, London Bridge, London SE1 1UL, UK. Correspondence and requests for materials should be addressed to E.J.B. (email: elizabeth.bradbury@kcl. ac.uk)
} 
extracellular factors which themselves actively inhibit restoration of function. These act acutely and chronically to prohibit compensatory changes in neurons which, perhaps if overcome, could transform the scar into a more effective repair process which both isolates damage and generates an environment in which injury could be surmountable.

Here we review recent literature regarding the composition and role of the spinal injury scar, including processes leading to scar formation and maintenance, the cellular and extracellular components of the scar, and how these interact with other mediators of tissue pathology. We discuss the complexities of the scar and its seemingly opposing roles, often classified in an overly simplistic manner as good or bad, and finally we discuss the potential for therapeutic targeting of the scar to achieve functional repair of the injured spinal cord.

\section{Scar formation and maintenance}

Tissue scarring in the CNS. Injury to any tissue results in a healing response, the purpose of which is firstly to curtail damage and restore homoeostasis and then, where possible, to restore tissue and organ function. Inflammation, tissue reformation (involving cell proliferation and/or migration) and tissue remodelling are conserved repair processes, although their success in restoring function varies across different tissues. A scar consists of the cells and extracellular matrix (ECM) formed as the result of attempted wound repair. In many organs, the formation of a scar is associated with a resolution phase and restoration of key functions of the tissue. The healed tissue may not directly recapitulate the pre-injury state but it regains some ability to execute its original function ${ }^{13}$. However, the process of tissue scarring in the CNS is more complex than for many other tissues, and is associated with chronic non-resolving pathology.

Disease and injury to the CNS is almost always accompanied by some degree of reactive gliosis, inflammation and scarring. Scar tissue and associated deposition of ECM molecules such as chondroitin sulfate proteoglycans (CSPGs, discussed in more detail below) has been reported in humans and experimental animal models following traumatic brain injury ${ }^{14}$ and stroke $e^{15}$, as well in neurodegenerative disorders such as Alzheimer's Disease $^{16}$, and disorders with a predominantly demyelinating and inflammatory pathology such as multiple sclerosis ${ }^{17,18}$. However, despite the occurrence of reactive tissue changes and scarring in several other CNS pathologies, spinal cord injury represents a particularly striking example where wound repair is inefficient and injury-induced pathological changes are insurmountable.

There are several likely contributing factors to these regional and injury-specific differences in CNS scarring. Cell types involved in scarring in different CNS regions are phenotypically different ${ }^{19}$. There are also differences in the levels of neuroinflammation and astrocyte activation after brain and spinal cord trauma, with increased expression of inflammatory cytokines and damage-exacerbating leukocytes ${ }^{20-22}$ and more abundant and widespread astrocytosis in spinal cord injuries compared to brain injuries $^{14,20}$. There are also differences in ECM composition and distribution between brain and spinal cord pathologies ${ }^{23-25}$. In this Review we specifically focus on scarring following spinal cord injury, and discuss the dynamic cellular and extracellular interactions that culminate in a hostile scar environment with limited capacity for repair.

Defining the spinal injury scar. The scar that forms after a spinal cord injury is generally considered to have two distinct components: the lesion core, which is primarily composed of stromalderived fibroblasts and inflammatory immune cells, and the lesion border, or penumbra, which surrounds the core and is primarily composed of hypertrophic astrocytes ${ }^{26-28}$. The term glial scar has historically been used to describe the astrocyte border component of the scar ${ }^{29-31}$, although some investigators use the term more widely to reflect the entire lesion including both glial and non-neural components ${ }^{32}$. Other distinctions have been made between the glial scar and the fibrotic scar ${ }^{27,33}$, and specific components of the lesion (the core, the astrocyte border, and surrounding tissue) have recently been referred to as lesionrelated tissue compartments ${ }^{31}$. While these distinctions are valid, and scar components undoubtedly become spatially compartmentalized in a chronic scar, these components are nonetheless interlinked, and evidence suggests that there may be temporal dependence and bi-directional cross talk between them. Furthermore, the scar should not be considered as an isolated component of spinal injury pathology since it is shaped by processes of inflammation and tissue and matrix remodelling. Here we use the term spinal injury scar, which encompasses both cellular and extracellular components across the lesion core, lesion border and surrounding penumbra (Fig. 1). Below, we discuss the events after the initial spinal cord trauma that contribute to spinal injury scar formation and maintenance.

Acute signalling events post-injury. The majority of spinal cord injuries feature a contusive component, where compromised spinal canal shape or volume causes physical deformation of spinal cord tissue ${ }^{2}$ (rarer presentations include sharp penetrating trauma to the spinal cord, or purely ischaemic lesions following vascular compromise). Tissue deformation transmits shearing and compressive forces on axons and blood vessels and initiates a cascade of pathological processes. Below, we discuss these processes, focusing on changes occurring immediately after the initial spinal cord trauma up to one day post injury. These acute postinjury events signal the beginning of the injury cascade, which culminates in chronic pathology and scarring (summarized in Fig. 2).

Vascular trauma leads to haemorrhage, and accumulating blood sera increases tissue colloid osmotic pressure, causing local oedema and swelling ${ }^{34}$. This damage, along with vasospasm of spared vessels, leads to tissue ischaemia. ATP released from damaged or metabolically-compromised cells acts on purinergic receptors to induce microglial chemotaxis towards the injury zone to protect against spread of damage ${ }^{35-38}$. ATP gradients are also propagated via connexins ${ }^{39}$. Oligodendrocytes, oligodendrocyte precursor cells (OPCs), microglia and astrocytes express a heterogeneous mix of $\mathrm{P} 2$ receptor subtypes, and respond reactively to increasing levels of ATP following trauma ${ }^{40,41}$.

Subsequently, tissue reperfusion induces further oxidative stress, glutamate release and death of neighbouring neurons and glia via excitotoxicity ${ }^{42}$. ATP release, dramatic loss of cellular and extracellular ionic homeostasis and excessive calcium levels results in the activation of calpains, phospholipase $\mathrm{A}_{2}$ and lipoxogenase. This is followed by the generation of bioactive lipid mediators and free radicals ${ }^{43}$. Progressive oxidation of fatty acids in cell membranes and myelin (lipid peroxidation) occurs. Furthermore there is feed-forward propagation of the injury and cell reactivity, as bioactive mediators potentiate ATPmediated calcium increases in glia ${ }^{40}$ and additional blood vessel endothelial cell damage results in an expanding zone of haemorrhagic necrosis ${ }^{44}$.

Concomitantly, haemostasis is the first stage of wound healing: endothelial cell trauma results in platelet adhesion and activation, the coagulation cascade, and thrombin-mediated conversion of fibrinogen to fibrin, to form a clot. The onset of hemostasis ${ }^{45}$ is associated with reactive changes in resident glia and represents a potent inflammatory stimulus. Platelets themselves are an 
a
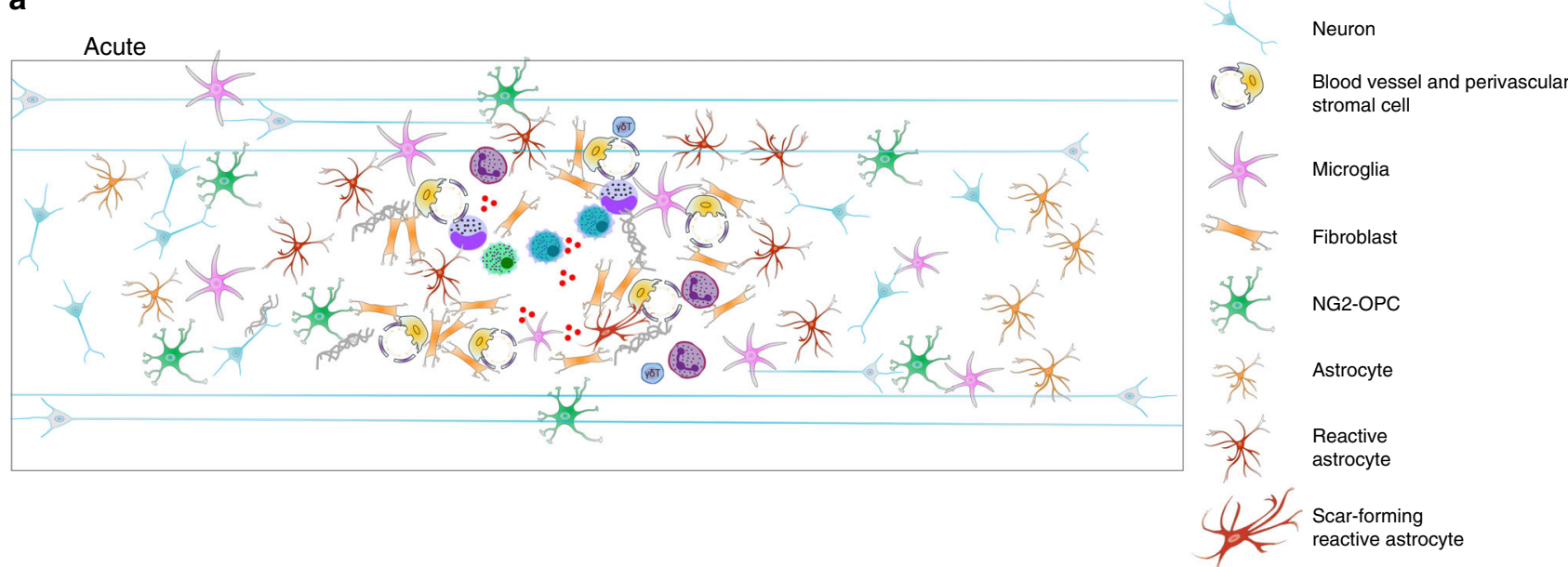

b Chronic

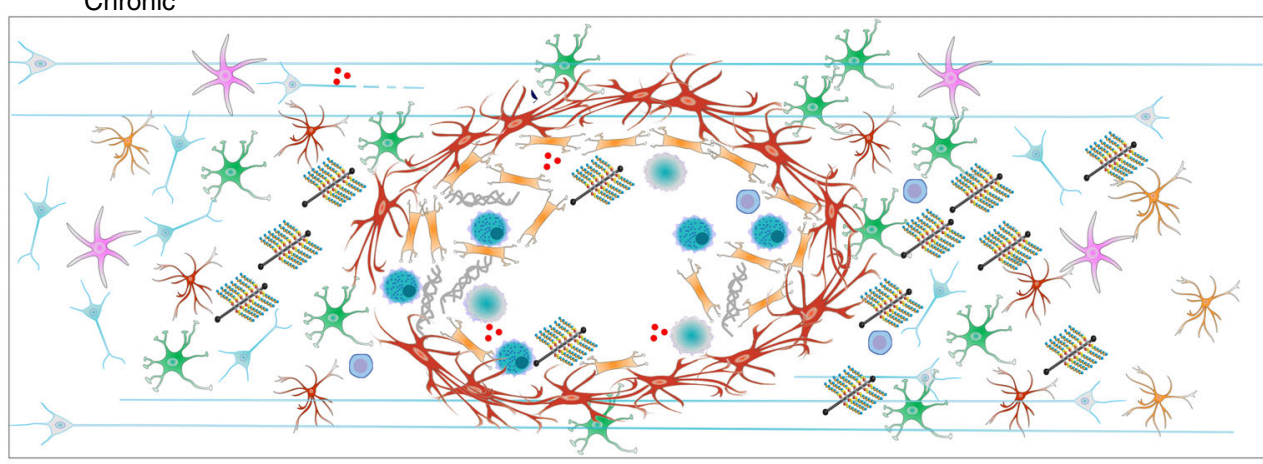

Neutrophil
"M1" macrophage
"foam cell"
Adaptive immune cells
$\begin{aligned} & \text { (type-1 collagen, periostin, } \\ & \text { fibronectin) }\end{aligned}$
$\therefore$ CSPG
DAMPs

Fig. 1 Cellular and extracellular composition of the spinal injury scar. Traumatic spinal cord injury triggers a complex cascade of events that culminate in the spinal injury scar which consists of multiple cell types as well as extracellular and non-neural components. a In the acute post-injury phase (0-72 $\mathrm{h}$ ), cell death and damage lead to release of a number of cellular and blood-derived DAMPs (damage associated molecular patterns). These are powerful activating and inflammatory stimuli for stromal cells, astrocytes, NG2 + OPCs and microglia. Fibroblast-like cells proliferate from perivascular origin. Activated cells increase deposition of extracellular matrix molecules such as chondroitin sulfate proteoglycans (CSPGs) and stromal-derived matrix. Circulating immune-responders (neutrophils, monocytes) are recruited, their relative expression of cytokines, chemokines and matrix metalloproteinases becomes shaped by the early injury environment, and a mixed immune cell phenotype (M1, pro-inflammatory; M2, pro-resolving) is initially adopted. This becomes increasingly proinflammatory. $\mathbf{b}$ In the chronic spinal injury scar, monocyte-derived macrophages/microglia adopt a predominantly M1 phenotype. Rather than entering a phase of resolution, responding innate immune cells present DAMPs to circulating adaptive immune cells and pathology spreads. Reactive astrocytes hypertrophy, upregulate expression of intermediate-filament associated proteins and secrete matrix CSPGs. Fibroblast-like cells contribute to fibrotic tissue remodelling and deposition of stromal-derived matrix. Innate immune cells become unable to process cellular and matrix debris effectively and become synonymous with lipid-rich foam cells. Scar-forming reactive astrocytes organise into a barrier-like structure which separates spared tissue from a central region of inflammation and fibrosis where wound-healing fails to undergo resolution. In most mammalian species a chronic cystic cavity develops. Wallerian degeneration of injured axonal projections contributes to continued extracellular deposition of axonal and myelin debris, which is ineffectively processed by immune cells, and along with CSPGs, acts to inhibit neuronal regeneration and neuroplasticity long-term

abundant source of inflammatory peptides and protein mediators and release cytokines, chemokines and eicosanoids which readily communicate with resident spinal cord cells and non-resident leukocytes. These signals cause rapid neutrophil infiltration within an hour (peaking within $24 \mathrm{~h}$ ), which secrete MMP9, a type IV collagenase which acts on basement membranes to further permeabilise the blood brain barrier ${ }^{34}$. Furthermore, cellular and extracellular factors (which are rapidly induced as a result of trauma, injury expansion and necrosis) constitute hostderived danger signals ${ }^{46}$. Damage associate molecular patterns (DAMPs) are sterile inflammatory stimuli (such as ATP, HMGB1, IL33) which activate prototypical pathogen recognition receptors (TLRs, NLRs, signalling via MAPK, NFKB) to induce secretion of proinflammatory cytokines and chemokines from both neurons and glia, compounding reactive gliosis and acting to recruit circulating immune responders ${ }^{47-50}$.

Non-resolving pathology. Non-resolving pathology results in incomplete tissue repair and formation of a scar. Each cell type that contributes to this pathology, and its respective phenotype, is inherently linked to the environment it finds itself in. This environment constitutes a milieu of other resident and nonresident cells, the signals they transmit and the biochemical and biophysical properties of the extracellular environment in which they reside. Below, we discuss cellular and extracellular changes occurring in the sub-acute period, from days to several weeks after the initial spinal cord trauma, and their contribution to the spinal injury scar. Cellular and extracellular components of the 


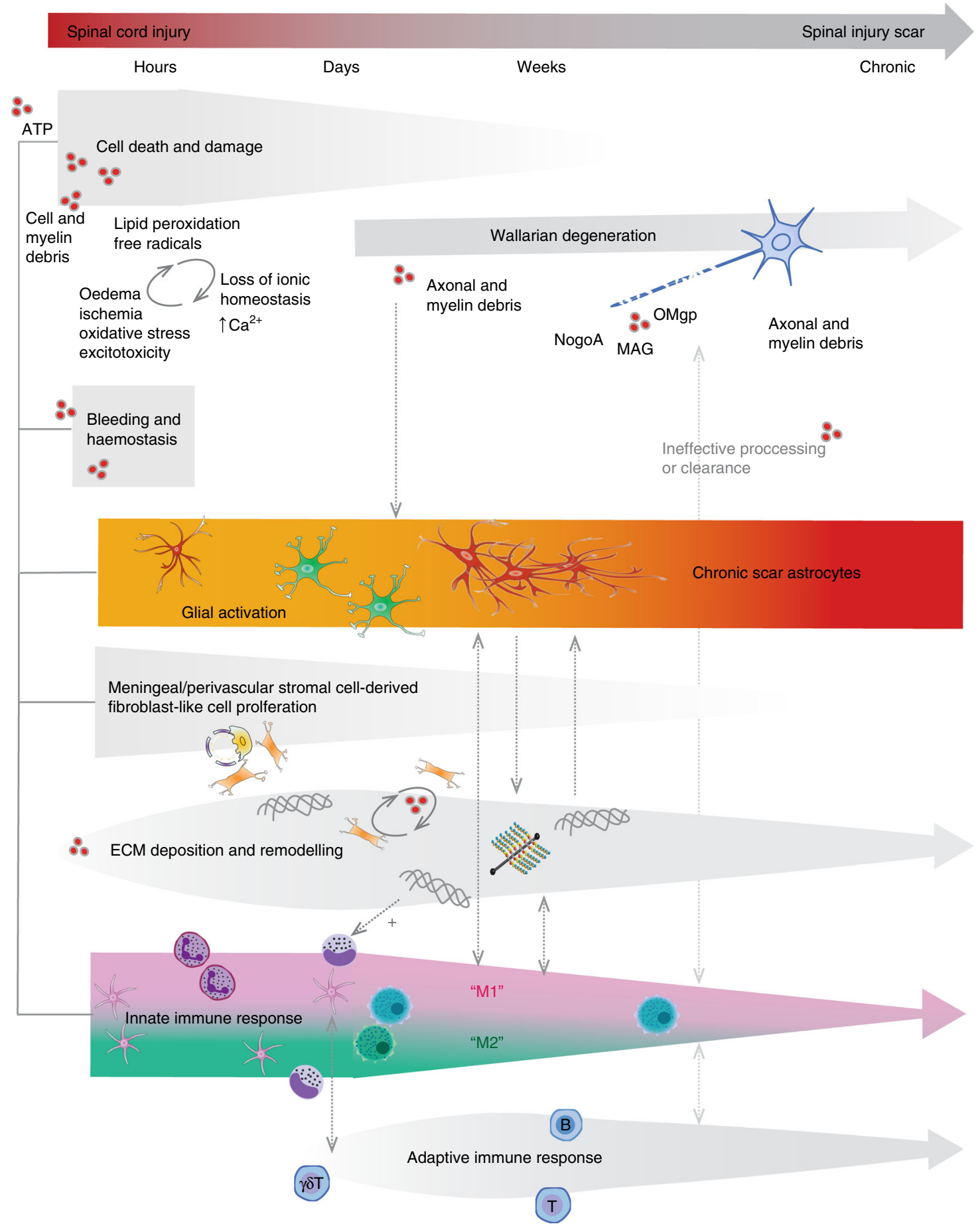

spinal injury scar in the acute and chronic phases of spinal cord injury are depicted in Fig. 1.

\section{Cellular components of the scar}

Astrocytes. Astrocytes become reactive following spinal cord injury. The degree of reactivity is influenced by a number of cellsurface receptors for DAMPS and proinflammatory cytokines and chemokines $^{51}$, and ranges from temporary changes in gene expression and cell morphology to significant hypertrophy, spatial rearrangement and proliferation (collectively termed astrogliosis $)^{52,53}$. Additionally, diverse astrocyte subsets and phenotypes may exist following spinal cord injury (Box 1).
Astrocytes are also known to dynamically switch from reactive to quiescent when transposed from injured to naïve spinal cord ${ }^{54}$. Reactive astrocytes densely populate the borders of the injury epicentre, hypertrophy and strongly upregulate the expression of intermediate filament proteins such as GFAP, nestin and vimentin $53,55,56$. This corresponds with elongation and extension of overlapping processes (unlike parallel and radial processes found throughout normal CNS tissue architecture) and the organization of astrocytes into a barrier-like structure ${ }^{57}$.

This barrier is reinforced by proliferation and organization of a local astrocyte population at the injury border, thought to be mediated via STAT-3 dependent signalling ${ }^{58,59}$ and leucine 
Fig. 2 From injury to scar: time course of progressive scar pathology showing interlinked relationships between different components of the spinal injury scar. Following traumatic spinal cord injury, acute cell death and damage triggers release of cell-derived and blood-derived DAMPs, ATP release, dysregulated ionic homeostasis oxidative stress and excitotoxicity, which represent potent stimuli for triggering glial cell activation, stromal cell proliferation, deposition of extracellular matrix (ECM), and recruitment of circulating innate immune cells. Within a few days following injury, monocytederived macrophage/microglia adopt a predominantly M1 phenotype which do not favour resolution and tissue remodelling becomes fibrotic. Proinflammatory innate responders also present DAMP-derived antigens (such as MBP) to T and B-cells. B cells, in turn, may present antigens to T-cells, triggering their expansion. During this time, reactive astrocytes proliferate, hypertrophy and overlap in order to isolate this zone of non-resolving pathology from spared tissue. They also secrete matrix CSPGs, which are known to downregulate neuronal plasticity. Wallerian degeneration of degenerating axonal tracts contributes to continued deposition of axonal and myelin debris, which is ineffectively processed by immune cells and leads to the deposition of myelin-associated molecules (MAG, Nogo, OMgp) which are known inhibitors of neuronal regrowth. Ongoing Wallerian degeneration at later post-injury stages further triggers gliosis and neuroinflammation. Dashed grey arrows show cross talk between different components of the spinal injury scar, which is usually bidirectional. For example, CSPGs released by reactive astrocytes are thought to activate receptors on macrophages/microglia to induce a proinflammatory phenotype and in turn increasing inflammation induces further astrocytic reactivity and CSPG deposition. Fibroblast-like cells also synthesise type 1 collagen, implicated in the induction of astrogliosis and further deposition of matrix molecules. Cross talk between the innate and adaptive immune response also propagates inflammatory pathology and further influences glial activation and CSPG production. The dynamic interactions between inflammation, dramatic tissue and ECM remodelling and reactive cellular and extracellular changes drive the progressive, propagating pathology that culminates in the spinal injury scar

\section{Box 1 | Phenotypic diversity and plasticity of astrocytes: emerging evidence from brain and spinal cord injury}

There is increasing data available on the cellular profile and phenotypic diversity of astrocytes after injury. Phenotypic nomenclature comparable to that adopted in characterization of macrophages/microglia has been used for the transcriptional profiling of cultured astrocytes isolated from the CNS under different injury conditions. Ischemic injury in the brain (modelling stroke) leads to a trophic A2 polarization state. By contrast, activated microglia from models of neurodegenerative and neuroinflammatory diseases and traumatic optic nerve crush injury release factors such as IL1 $\alpha$, TNF and complement component subunit $1 \mathrm{q}(\mathrm{C} 1 \mathrm{q})$ which induce a neurotoxic state in $\mathrm{A} 1$ astrocytes 55,94 . In the optic nerve, there is evidence that axotomy-induced $\mathrm{A} 1$ astrocytes, in turn, kill axotomized neurons. In these studies, microglial-derived mediators were necessary and sufficient to induce an A1 phenotype. Whether this occurs following spinal cord injury is, as yet, unreported.

Genetic profiling of reactive astrocytes and scar-forming astrocytes following spinal cord injury (isolated by laser-capture microdissection at 7 and 14 days post injury, respectively) has recently been reported. Reactive astrocytes were associated with selective upregulation of Nes, Ctnnb1, Axin2, Plaur, Mmp2, and Mmp13 whereas scar-forming astrocytes selectively upregulated Cdh2, Sox9, Xylt1, Chst11, Csgalnact1, Acan, Pcan and Slit2 54 . Furthermore, using these genes as population markers, FACs isolated nestin-GFP+ reactive astrocytes were found to convert to a naïve phenotype following transplantation into uninjured tissue but become scar-forming when transplanted into injured tissue, an effect thought to be mediated by a $\mathrm{N}$ cadherin-dependent interaction with type 1 collagen ${ }^{54}$. Thus, astrocytes are able to display phenotypic plasticity, and tissue environment is a crucial influence over cellular behaviour.

The GFAP-RiboTag mouse can be used to perform high-throughput RNA sequencing on astrocytes following injury and probe the effect of particular genes in the astrocyte response ${ }^{108}$. Two weeks after a spinal crush injury RNA-seq revealed differential expression of over 6000 genes in astrocytes, changes described as congruent with prior transcriptomic analysis following ischemic stroke lesion 55,108 . However, whether the astrocytes sampled here represent A2-like trophic astrocytes has not been ascertained.

There are some discrepancies between gene expression findings using these different methodologies. For example, of the eight genes associated with scar-forming astrocytes isolated using laser capture microdissection 54 , the RNA-seq dataset only supports increased expression in one of these $(X y / t 7)^{108}$, whereas four of the six genes ascribed to reactive astrocytes ${ }^{54}$, are increased (Nes, Axin2, Plaur, Mmp2) ${ }^{108}$. Spatial differences in sample selection may contribute to these disparities. Techniques such as 3D intact-tissue RNA sequencing 182 may overcome this problem in the future. Indeed, further characterization of spatio-temporal phenotypic diversity and plasticity would aid research into how astrocyte phenotype and scar progression may be modified by changes to matrix components or perturbation of the immune response. Such methods, alongside new purification techniques for in vitro analysis, will likely provide increasingly nuanced understanding of the relationship between astrocytes, microglia, non-resident immune cells and the tissue environment ${ }^{79,185}$.

zipper-bearing kinase (LZK, MAP3K13) expression ${ }^{56}$. Ependymal cell-derived astrocyte-like progeny contribute to this population of astrocytes in certain spinal injury models ${ }^{60}$, athough evidence suggests this contribution is minor following contusive injuries ${ }^{61}$. Tight linking of astrocytes at the injury borders is associated with reformation of the glia limitans and the containment of immune cells and fibroblast-like cells within the injury epicenter, via ephrin-mediated cellular adhesion ${ }^{62,63}$. Thus, a population of reactive astrocytes act to spatially isolate damage and fibrosis from spared tissue.

An overlapping population of astrocytes are further associated with the chronic maintenance of this structure. These are referred to as scar forming astrocytes ${ }^{64}$. Though astrocytes alone are not responsible for the formation of a scar, they are major cellular players activated and maintained during post-injury pathology, inflammation and tissue and matrix remodelling. Alongside other cells and extracellular factors, astrocytes shape the scar cellular and extracellular milieu sub-acutely and chronically.

Fibroblast-like cells. Fibroblasts are ubiquitous in peripheral connective tissues and organs and are the principal generators of stroma, including the ECM. By contrast, under normal conditions within the CNS, fibroblast-like cells are mostly associated with the vasculature, and contribute only to the basal laminae. However, injury to the spinal cord induces a significant fibroblast response which produces matrix components. These matrix components may inhibit neural regeneration directly, and promote prolonged tissue remodelling via interaction with inflammatory cells (detailed below). These stromal elements become spatially 
compartmentalized by surrounding reactive astrocytes to form the fibrotic core of the spinal injury scar.

Fibroblasts proliferate from meningeal cells if the dura is compromised $^{27,33}$ and can derive from perivascular cells in rats ${ }^{65}$ and mice ${ }^{63}$ following contusion injury. There is some evidence that fibroblast-like stromal cells derive from pericytes; the PDGFR $\beta^{+}$Glast $^{+}$perivascular cell population, termed type A pericytes, proliferate in response to injury and contribute to fibrotic scarring ${ }^{66}$. Preventing Glast $1+$ cell proliferation leads to failure of wound sealing, exacerbated lesion volume and decreased matrix deposition ${ }^{66}$, whereas moderate reduction of pericyte-derived fibrosis was found to reduce scar pathology and confer functional recovery ${ }^{67}$. However, whether this is a truly separate population or whether it overlaps with known cell types (such as Glast+ astrocytes in the glia limitans) is unclear.

An increase in type-1 pericytes (distinct from those described above) has also been described, following a non-contusive dorsal funiculus lesion using a nestin-GFP/NG2-DsRed transgenic mouse line ${ }^{68}$. However, NG2 is also a marker of OPCs, Schwann cells and macrophages following spinal cord injury ${ }^{69}$. Thus, there is some ambiguity as to the response of pericytes following injury, although collectively there is evidence that fibroblast-like cells are derived from a perivascular PDGFR $\beta+$ origin $^{63,66,70}$.

Oligodendrocyte precursor cells. NG2+ OPCs become reactive after spinal cord injury, have a significant proliferative capacity and are spatially intermingled with other reactive glia at the injury border ${ }^{71,72}$. Two main contributions of OPCs within the spinal injury scar environment have been described, with seemingly opposing roles. OPCs contribute to remyelination, either by oligodendrogenesis or through differentiation into remyelinating Schwann cells ${ }^{73-75}$, and also hypertrophy and increase expression of NG2, a proteoglycan thought to mediate entrapment of neurons ${ }^{76}$. However, the roles of OPCs in the spinal injury scar have been obscured experimentally by overlap of markers with other cells. Alongside NG2, PDGFRa, two ganglioside antigens, and a cyclic nucleotide phosphodiesterase (also thought to be expressed in microglia), OPCs also express the traditional astrocyte marker GFAP, and may differentiate into a de novo population of astrocytes in the scar ${ }^{72,77}$. Additionally, both PDGFRa and NG2 are also thought to be expressed by at least one type of perivascular/pericyte-type cell ${ }^{71}$. The use of fate mapping transgenic mouse lines, such as those expressing Cre recombinase under control of the PDGFRa promoter/enhancers $^{73-75,78}$, alongside inclusion/exclusion of specific markers, will further define their role and contribution to scarring after spinal cord injury.

Resident microglia and innate and adaptive immune cells. By $24 \mathrm{~h}$ after spinal cord injury, blood-derived monocytes are recruited into the lesion. Upon extravasation, DAMPs and the associated reactive and inflammatory environment shape their differentiation phenotype. Meanwhile, resident microglia retract cellular processes and become morphologically indistinguishable from infiltrated monocyte-derived macrophages. Until recently, microglia and macrophages were only distinguishable using relative gene expression of CD45 (macrophages being defined as $\mathrm{CD} 11 \mathrm{~b}^{+}, \mathrm{CD} 45^{\text {high }}$ and microglia $\mathrm{CD} 11 \mathrm{~b}^{+}, \mathrm{CD} 45^{\text {low }}$ ) or chimeric models. Specific, or enriched, markers for microglia have now been discovered, including transmembrane protein 119 $(\text { Tmem } 119)^{79}$, P2ry12 and Fc receptor-like S (FCRLS) ${ }^{80}$. Tracking resident microglia via a genetic strategy has shown that spared microglia proliferate and repopulate the lesion core alongside monocyte-derived macrophages ${ }^{38}$ and recent availability of
Tmem119 reporter mice ${ }^{81,82}$ will further elucidate the role of microglia in spinal injury scarring.

A monocyte-derived or microglia-derived macrophage phenotypic spectrum exists, from pro-inflammatory (termed M1, secreting TNFa, IL-1 $\beta$, IL-6, IL-12) to pro-repair (termed M2, secreting IL-10, IL13). Following spinal cord injury, there is initially a mixed M1/M2 response ${ }^{83}$. The release of proinflammatory cytokines at the injury site further mobilizes resident and blood-derived cells to phagocytose debris ${ }^{84,85}$ and affects the phenotype of other nearby resident cells.

The adaptive immune system also plays a role. After spinal cord injury, the recruitment of $\gamma \delta \mathrm{T}$ cells, and production of proinflammatory IFN $\gamma$ occurs within $24 \mathrm{~h}$ following injury ${ }^{86}$. Other adaptive immune system components are recruited by 7 days, and contribute to non-resolving trauma-induced autoimmunity ${ }^{87}$. Leukocytes present DAMP-derived antigens (such as MBP) to T and B-cells. B cells, in turn, may present antigens to Tcells, triggering their expansion. Furthermore, B-cells differentiate into plasma cells synthesizing auto-antibodies, further fuelling a feed-forward immune response ${ }^{88}$.

Unlike conditions in which successful wound healing occurs, there is no effective resolution to cellular recruitment and inflammation after spinal cord injury. Monocyte or microgliaderived macrophages remain in the injured spinal cord indefinitely ${ }^{89}$. Macrophages maintain $45 \%$ of peak activation months after injury 90 and their phenotype does not undergo the switch from pro-inflammatory to pro-repair associated with the next phases of wound-healing in other organ tissues ${ }^{91}$. The early arginase $1+(\mathrm{M} 2$-like) differentiating infiltrating population is not maintained $^{83}$ and the spectrum of innate immune cell activation phenotype is predominately M1 polarized. Adaptive immunity is non-resolving, whereby lymphocytes remain indefinitely in the spinal injury scar.

Interaction between cell types. The response of astrocytes, OPCs, microglia and infiltrating innate and adaptive immune cells is continually influenced by one another and the tissue environment (Fig. 2 depicts the time course of reactive resident and nonresident cell recruitment and activation, and their cross-linked interactions which lead to the chronic spinal injury scar). There are multiple direct and indirect cellular interactions mediated by cytokines and chemokines which underlie this (alongside interactions which occur via ECM components, discussed below). Perivascular astrocyte endfeet are an integral part of the endothelial blood spinal cord barrier, and thus astrocytes are in a position to regulate the magnitude of leucocyte recruitment. Astrocyte expression of Socs $3^{92}$ or $\mathrm{NFKB}^{93}$ increases monocyte infiltration to the lesion epicentre. In addition, resident and infiltrated microglia/macrophages express a number of receptors for proinflammatory chemokines and cytokines released by reactive astrocytes (such as IL-6, IL1 $\beta$, CCL2), contributing to a cell signalling environment which potentiates M1 polarization. In turn, astrocytes express receptors for a number of inflammatory mediators released by immune cells (including IFN $\gamma$, IL6, IL1 $\beta$, $\mathrm{TNF} \alpha$, inducing extensive astrocyte reactivity and astrogliosis $^{53,94}$. Thus, there is an intimate link between astrocytes and resident and infiltrating immune cells during formation of the spinal injury scar. Astrocyte-fibroblast interactions have been shown to spatially compartmentalize the fibrotic core ${ }^{62}$ and recent evidence suggests that microglia may provide an additional interface between these cells, which the authors term the microglial scar ${ }^{38}$.

In addition to direct cellular cross-talk, almost all parenchymal cells express receptors for a multitude of signalling molecules present in the external injury microenvironment and indirectly 
affect cell activation and phenotype of surrounding cells. Within this, there are canonical regulators. For example, abolishing Wnt signalling in OPCs has been shown to reduce monocyte accumulation and astrocyte hypertrophy ${ }^{95}$. Furthermore, neurons themselves are directly contacted by cells in a manner which inhibits reestablishment of neuronal connectivity. Proinflammatory $\mathrm{ED} 1 / \mathrm{CD} 68+$ macrophages induce axonal dieback upon contact $^{96,97}, \mathrm{NG} 2+$ OPCs mediate neuronal entrapment ${ }^{76}$, and perivascular Glast $1+$ cells are also directly contacted by stalled axons ${ }^{67}$.

Importantly, the reactive cellular responses after spinal cord injury are not effectively resolved and many aspects are maintained chronically. Macrophages retain activity long-term with maintained M1 like characteristics. Glia continue to be reactive in regions of tissue spared by injury and in areas remote from the site of trauma ${ }^{98}$, partly in response to Wallerian Degeneration ${ }^{99,100}$. Proximal to the lesion, glia remain hypertrophic, forming a compacted astroglial scar border in which spared tissue is permanently isolated from a zone of unresolved pathology, fibrosis and tissue loss. This zone is not effectively repopulated by neurons or glia and, in most mammalian species, develops into a chronic cystic cavity (Fig. 1).

\section{Extracellular components of the scar}

The CNS ECM is rich in glycoproteins and proteoglycans. Hyaluronan forms a backbone for the attachment of tenascins and sulphated proteoglycans, stabilised by link proteins. This is arranged either diffusely in the interstitial space or more densely assembled around the cell soma of particular neuronal subtypes (as perineuronal nets), or around axonal nodes of ranvier or synaptic boutons. These structures confer neural stability, localizing molecules such as CSPGs, which effectively restrict largescale plasticity following a critical period in development ${ }^{101}$. Following injury, resident glia and stromal cells, which do not normally contribute parenchymal matrix, begin to contribute matrix components, and extracellular DAMPs are present in both sub-acute and chronic phases.

A vast number of ECM molecules undergo differential regulation following spinal cord injury (for a large scale validation see ref. ${ }^{50}$ ) and many of these play a role in neuroprotection or spontaneous repair and are not refractory to recovery. Fibrous matrix forms a seal or tissue bridge between retracting lesioned parenchyma. This is particularly apparent in injuries where spinal tissue is rendered non-continuous, for example, following hemisection or transection. Fibroblast-derived collagenous matrix is also a major component of the $\mathrm{ECM}^{33}$ (and in vivo ablation of fibroblasts compromises tissue integrity following injury $)^{66}$. Basal laminae is restored via matrix deposition of collagen VI, nidogen, fibronectin and laminin, which are traditionally neuronal-growth permissive molecules. However, in the context of spinal cord injury, such ECM molecules are also implicated in pathological tissue remodelling or inflammation. For example, fibronectin, matrix glycoprotein tenascin $\mathrm{C}$ and hyaluronan fragments also act as endogenous TLR ligands and represent DAMPs ${ }^{48}$.

Extracellular components fuel fibrosis and scarring. Initial and expanding secondary pathology generates a large amount of cellular and myelin debris, sustained by longer-term Wallerian degeneration, oligodendrocyte apoptosis and demyelination. The presence of debris, and its breakdown products, supports an ongoing foam-cell-like macrophage phenotype ${ }^{102}$ where ineffective phagocytosis and lipid processing means extracellular stimuli are maintained ${ }^{85}$ and are presented to adaptive immune cells, which contributes to a non-resolving auto-immune response to injury ${ }^{87}$. Thus, the extracellular environment is undergoing both chronic inflammation and glial reactivity, associated with aberrant tissue remodelling and matrix deposition. There is increasing understanding as to how these processes are intertwined.

A recent study demonstrated that perivascular PDGFR $\beta+$ cells (described as pericytes) upregulate expression of the ECM molecule periostin, which in turn upregulates TNF $\alpha$ expression from infiltrating monocyte-macrophages and leads to proliferation of PDGFR $\beta$ + cells, type-I collagen deposition and fibrosis ${ }^{70}$. Perivascular-derived type-1 collagen has also recently been implicated in linking fibrosis and astrogliosis, where an Ncadherin dependent interaction between extracellular type-1 collagen and astrocytes was found to induce scar-forming astrogliosis in mice ${ }^{54}$. Thus, there is expanding evidence supporting a role for perivascular Colla1-cell derived fibrotic matrix following contusion injury in mice ${ }^{63}$. PDGFR $\beta+$, fibronectin-rich fibrotic matrix deposition is also observed in rats in the peripheral rim of the cavity and outlining blood vessels ${ }^{103}$, suggesting a somewhat conserved contribution despite differences in cavity formation between mice and rats. Matrix deposition of type-1 collagen has also been described as a perivascular-fibroblast-derived scaffold for neoangiogenesis ${ }^{65}$. Defining the role of collagen in fibrosis and angiogenesis in spinal cord injury requires further study.

Extracellular components are inhibitory to neural regeneration and plasticity. In addition to an ongoing DAMP role for myelin debris, myelin-associated molecules confer extrinsic inhibition to neurons. These include Nogo A, myelin-associated glycoprotein (MAG) and oligodendrocyte myelin glycoprotein (OMgp). NogoA is a potent inhibitor to neural plasticity and regeneration following spinal cord injury ${ }^{104}$, preventing axons from overcoming the spinal injury scar environment. Transmembrane receptor complexes are identified, converging on the canonical RhoA/ ROCK signalling pathway, resulting in destabilization of the actin cytoskeleton and local arrest and collapse of growth cones ${ }^{105}$. Furthermore, CSPGs (Box 2) are upregulated by reactive glia following spinal cord injury both perilesionally and at distal spinal segments ${ }^{98,106}$ and are associated with decreased plasticity and abortive regeneration ${ }^{107}$. Recent evidence suggests that scarforming astrocytes express brevican, and NG2, though not aggrecan ${ }^{108}$. There is some evidence that core CSPG proteins are inhibitory to neuronal growth ${ }^{109}$ but CS-GAG chains are known to confer significant inhibition following injury as their removal promotes anatomical and functional recovery following spinal cord injury ${ }^{110}$. Membrane-bound receptors to CS-GAGs, reported to mediate inhibition, include RPTP $\sigma^{111,112}$, leukocyte common antigen-related phosphatase (LAR) ${ }^{113}, \mathrm{NgR} 1$ and NgR3 ${ }^{114}$. Signalling pathways implicated have convergence with those of Nogo and other myelin inhibitors and include the Rho/ROCK pathway, activation of which is partly via PKC 115 and EGFR ${ }^{116}$ and coupled to Akt/GSK-3 activation ${ }^{117}$. CSPGs are also thought to inactivate neural intergrins ${ }^{118}$ and localise upregulated inhibitory guidance molecules such as semaphorin $3 \mathrm{~A}^{119}$. Thus, the injured spinal cord extracellular environment contains molecules which restrict neurite outgrowth and plasticity, and these are further upregulated and concentrated in the spinal injury scar and represent therapeutic targets ${ }^{120}$ (discussed below).

\section{Biomechanical properties of the scar}

Relatively little attention has been given to how the biomechanical environment of the injured spinal cord affects repair ${ }^{121}$. Cells are highly mechanosensitive and changes in the elastic properties of the environment alone can induce differentiation and migration ${ }^{122}$, and during development mechanical gradients guide axon pathfinding ${ }^{123}$. Astrocytes that are cultured on less 


\section{Box 2 | Structure of chondroitin sulfate proteoglycans (CSPGs)}

CSPGs are proteoglycans (PGs) consisting of a core protein with at least one covalently attached chondroitin sulfate glycosaminoglycan (CS-GAG) side chain (see figure). CSPG subtypes most commonly studied with respect to the inhibitory CNS environment include lecticans (aggrecan, versican, neurocan and brevican), the transmembrane protein NG2, phosphacan (transmembrane or soluble) and the small leucine-rich proteoglycans decorin and biglycan. There are also multiple less-well studied CSPGs revealed by proteomics analysis of scar matrix ${ }^{50}$. Lecticans are the most abundant CSPGs in the spinal injury scar and also feature globular domains: the G1 N-terminal domain and G3 C-terminal domains are important in their interaction via link-protein with hyaluronan (the backbone glycoprotein of the CNS matrix) and also tenascin, thus they are involved in matrix crosslinking. Core PGs undergo post-translational modification in the endoplasmic reticulum and golgi, catalysed by a number of enzymes. At particular serine residues a tetrasaccharide linking region is formed by sequential addition of xylose by xylosyl transferase, two galactose molecules by $\beta 1,4-G a l a c t o s y l t r a n s f e r a s e-I$

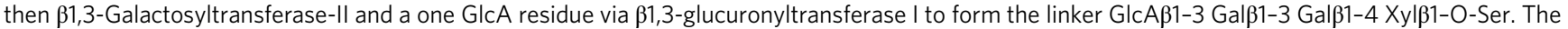
following addition of GalNac by a GalNac transferase I is crucial to initiate synthesis of the chondroitin sulfate backbone. If, at this point, Nacetylglucosamine (GlcNac) is added rather than $\mathrm{GalNac}$, synthesis of the heparan sulfate backbone is initiated ${ }^{186}$. CS-GAG chain polymerisation is the process by which alternating residues of GalNac and GlcA are then added to the proteoglycan linker region by the alternating activity of a GlcA transferase II and a GalNac transferase II. There are six actual enzymes identified which confer this glycosyltransferase activity. Alongside CS-GalNac transferase I and II, combinations of enzyme complexes of chondroitin synthase 1, 2 and 3 and chondroitin polymerising factor (ChPF) mediate GAG polymerization.
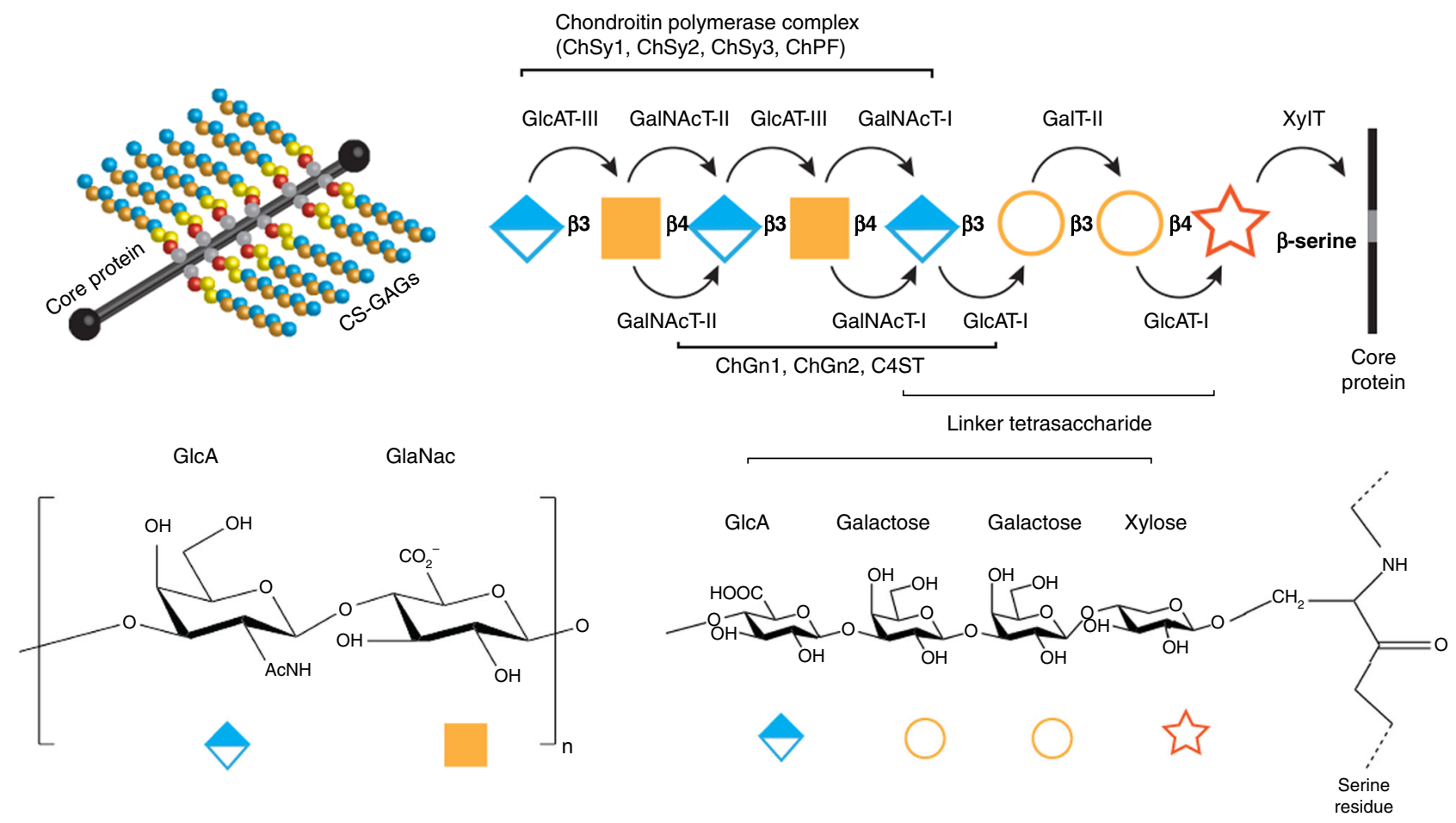

compliant, stiff substrates anatomically resemble reactive astrocytes, displaying hypertrophy and elongation, with stiffness of CNS implants correlating with induction of reactive astrocytosis ${ }^{124}$. Integrin-mediated links between fibrillar type 1 collagen (known to be stiffer in other tissues) and astrocyte reactivity are emerging ${ }^{54}$, which may be influenced by mechanotransduction. Atomic force microscopy has been used to characterize the spatiotemporal elastic stiffness properties of spinal cord tissue over 1 to 3 weeks following dorsal column crush lesion ${ }^{125}$. At these early post-injury time points, tissue softened in areas corresponding to scarring and ECM deposition. This was somewhat surprising because scar tissue outside the CNS is typically stiffer than surrounding healthy tissue ${ }^{126}$, and was attributed to a lack of collagen-1 and loss of CNS myelin in these types of injuries (both of which scale with tissue stiffness) ${ }^{127,128}$, as well as the cellular composition of the scar (glial cells are softer than peripheral scar myofibroblasts). It will be important to further characterize these properties in more clinically relevant contusion-type injuries and in chronic injuries with established scar tissue, particularly given the increasing evidence of a role for collagen- 1 in chronic contusive injuries ${ }^{54,63,129}$.

\section{The semantics of defining the scar as good or bad}

There has been some recent debate in the field on whether the scar is good or bad in terms of recovery from injury 108,130-132. We propose that these two different viewpoints reflect different interpretations of data which is, in fact, largely in agreement. Both historical and newer findings support a long-established principle, that the spinal injury scar performs dual, and seemingly opposing, roles; to protect tissue, and to inhibit repair.

As previously introduced, the classical description of the spinal injury scar is one which considered the astrocyte-rich injury 
border alone, termed the glial scar. Early observations of dense glial reactivity at the site of CNS lesions led to the hypothesis that the astrocytic scar inhibits axon regeneration, perhaps by forming an impenetrable barrier to axonal extension ${ }^{30}$. An inhibitory role for the scar and scar-associated molecules has been well documented ever since (as discussed above and $^{26,27,106,107,110,111,116,133,134}$ ). However, it has also long been acknowledged that the astrocytic scar has an important protective role in enabling the separation of healthy tissue from pathology following injury ${ }^{135-137}$. Thus, for decades the dual notions that the scar is associated with failed axonal re-connectivity (inhibitory) and also involved in a wound-healing response (protective), have existed.

In addition, as discussed above, there is now increased appreciation of the multiple cell types, beyond astrocytes, which contribute to spinal injury scarring, together with extracellular and non-neural components. This renders the term glial scar an insufficient descriptor. The multifaceted nature of the scar should be considered when interpreting experimental approaches which prevent scar formation. For example, a number of transgenic loss of function experiments have been performed to specifically investigate the role of astrocytes following spinal cord injury, including formation of the glia limitans and continued presence in the chronic scar. Early evidence suggested that double knockout mice for the intermediate filament proteins GFAP and vimentin (but not either protein alone) develop a less dense glial scar, with greater haemorrhaging, fibrosis and presence of debris following lesion to the dorsal funiculus ${ }^{138}$. Similarly, conditional ablation of proliferating (scar-forming) reactive astrocytes following injury increases edema, inflammation, oligodendrocyte death, tissue loss, demyelination and functional deficits ${ }^{139,140}$. Furthermore, deletion of SOCS3 or STAT3 in astrocytes results in lesion expansion, cell death and exacerbated functional outcome ${ }^{58,92}$. Thus, a number of studies have provided evidence for the importance of reactive astrocytes in preventing expansion of pathology into spared perilesional regions of the spinal cord. A recent extension of these studies utilized these deletion strategies to specifically assess the effects of reactive astrocytes on axonal regeneration ${ }^{108}$. The study revealed that spontaneous regrowth of damaged axons does not occur across a spinal crush injury following attenuation or ablation of scar forming astrocytes, despite boosting the regenerative state of these axons with a conditioning lesion and neurotrophin delivery ${ }^{108}$. This study claimed to reveal a new (and controversial) role of the astrocytic scar as being proregenerative. Our interpretation of these findings, however, is that they are largely consistent with previous studies ${ }^{58,92,140}$. A lack of axon regeneration after ablating reactive scar astrocytes does not necessarily mean that the glial scar aids axon regeneration. An alternative interpretation is that the regenerative boost afforded by conditioning lesions and neurotrophin delivery is not sufficient to overcome the prior-established lesion-exacerbating effects of preventing astrocytic scar formation. Instead, injured axons are presented with neural and non-neural components of the spinal injury scar, including NG2+ OPCs, inflammatory cells and CSPGs, all of which are known blockers of regeneration $110,111,116,133,141$. Indeed, greater dieback of axons from the injury site was observed in this study ${ }^{108}$, in line with an advancing wall of inhibitory factors no longer contained in the fibrotic lesion core. Thus, rather than overturning an old dogma, this study used elegant genetic tools to demonstrate an important role for scar-forming astrocytes in tissue protection following traumatic spinal cord injury, supporting previous observations ${ }^{58,92,139,140}$ and confirming the early hypothesis postulated by Gopal D. Das: "If, by some means, glial scar formation could be completely eliminated, most of the atrophying axons still might not show regeneration, and the spinal cord would be continuously invaded by loose connective tissue and other foreign materials and organisms while undergoing a protracted degeneration"135.

Although astrocyte components of the spinal injury scar are well evidenced to be beneficial initially, they (and other scar elements) have been suggested to be detrimental at chronic postinjury stages $32,142,143$. However, astrocyte ablation at chronic timepoints indicates that astrocytes themselves are necessary for maintaining tissue integrity in chronic injuries, as functional outcome is negatively affected by their removal 5 weeks post injury ${ }^{108}$. Though their contribution to extracellular signalling may have both beneficial and detrimental elements.Thus, it will be important to further characterise the molecular profile of the spinal injury scar at precise time points after injury, both for the cell types involved in scarring (e.g. Box 1) as well as the extracellular components. For example, recent proteomics studies of subacute (1-2 week) and chronic (8 week) spinal injury tissues have revealed differential expression of multiple growth factors and inhibitory and permissive ECM molecules at different postinjury stages ${ }^{144,50}$, requiring further study as to their specific roles in shaping the response to injury.

Thus, the dual nature of the spinal injury scar has long been known, yet continues to be reviewed, revisited and reinterpreted $19,64,92,108,132,142,143,145$. Rather than focus on good versus bad, perhaps efforts would be best directed at understanding and targeting specific aspects of the scar to aid recovery. For example, it may be beneficial to target components of the scar which are non-permissive to regeneration or plasticity rather than removal of the astrocytes themselves, even at chronic time-points. Targeting diverse cell types and phenotypes, as well as extracellular and non-neural components should also be considered, as well as the timings of such interventions. These approaches will be discussed in the following section.

\section{Therapeutic strategies}

Current experimental approaches for targeting the spinal injury scar attempt to either reduce scar formation, or to block inhibitory molecules associated with the scar, using a variety of surgical, pharmacological and genetic approaches. Some of these show promise for application in the clinic.

Attenuating scar formation. Although preventing formation of the astrocytic component of the spinal injury scar impacts negatively on wound-healing (discussed above), as the mechanistic understanding of spinal injury scar pathology increases, a number of studies in preclinical rodent models have targeted mesenchymal or fibrotic-derived components in a bid to limit amplification of tissue damage. On a gross tissue scale, if the dura is breached, dural apposition and/or patching with another soft tissue material (duraplasty) is suggested to limit fibrotic and connective tissue deposition from meningeal-derived fibroblasts. Decompressive durotomy followed by dural allograft has been shown in rodent models to reduce scar formation and lesion volume, but if the dura is not replaced, lesion volume increases dramatically ${ }^{146}$ and indeed expansion duroplasty is performed alongside decompressive durototomy in clinical evaulations ${ }^{147}$.

The fibrotic components of the scar can also be targeted pharmacologically. Systemic administration of the microtubule stabilizing antimitotic agents taxol or Epothilone B leads to reduced migration of scar-forming fibroblasts and suppression of extensive scar formation, enabling axon regeneration and functional recovery ${ }^{11,148}$. This highlights the potential for repurposing of epothilones and taxanes that are already used in cancer treatment ${ }^{149}$. Inhibiting collagen synthesis using the iron chelators 
BPY-DCA (which inhibits prolyl 4-hydroxylase, a key enzyme of collagen IV synthesis) and cyclic adenosine monophosphate (cAMP, which inhibits meningeal fibroblast proliferation) ${ }^{150,151}$ reduces fibrotic scarring and promotes neuroprotection and longdistance axon regeneration ${ }^{150}$. Treatment with clinically approved ion chelator deferoxamine and inhibition of lysl oxidase, another key collagen biosynthetic enzyme, also improves outcome after partial spinal transection injuries in rodents ${ }^{152,153}$.

The gene expression profile or phenotype of astrocytes may also represent potential therapeutic targets with modulating effects on scar formation. For example, selective inhibition of NFKB signalling in astrocytes reduces inflammation and is proreparatory in mice expressing a dominant negative $\mathrm{KB} \alpha$ under the GFAP promoter ${ }^{93}$. However, this is difficult to target therapeutically. If current studies of astrocyte phenotype in the mouse brain (Box 1) translate experimentally to the injured spinal cord, newly identified gene/signalling targets and matrix targets (below) could be manipulated to depress propagating scar pathology. In vitro, application of human recombinant TGF $\beta 3$ (but not the removal of mediators IL1 $\alpha$, TNF and C1q) was able to rapidly reverse transformation from an A1 phenotype to an unreactive status $^{94}$. Following optic nerve crush, this phenotypic conversion was demonstrated in vivo by delivery of antibodies to IL1 $\alpha$, TNF and $\mathrm{C}_{1} \mathrm{q}^{94}$. Manipulation of TGF $\beta 3$ was not reported in vivo, however multiple studies have targeted TGF $\beta 1 \& 2$ following spinal cord injury ${ }^{154}$ to reduce spinal injury scar formation, so it would be interesting to know whether this is a TGF 33 -specific effect. Indeed TGF $\beta 1$ and 2 are known to exert opposing effects to TGF $\beta 3$ on wound healing outside of the CNS, and human recombinant TGF $\beta 3$ has been utilized in clinical trials to promote dermal wound healing and scar reduction ${ }^{155}$.

Targeting the extracellular matrix. Following spinal cord injury, the extracellular environment contains molecules which interact directly with neurons and other cell types (discussed above). Some of these are thought to augment pathology and extent of spinal injury scarring, while some directly inhibit the ability of neurons to overcome a scar environment and generate novel connectivity. Both are potential therapeutic targets.

Periostin is a secreted ECM protein which has recently been implicated in contributing to scar formation, via propagating fibrosis and inflammatory signalling ${ }^{50,70}$. Daily intraperitoneal injections with a mouse monoclonal antibody against periostin from 4 days to 2 weeks after injury was shown to reduce the extent of tissue pathology and scarring, which led to functional improvements in sensorimotor tasks following contusion injury in mice $^{70}$. Generation of recombinant anti-periostin will allow this promising strategy to be further tested. Similarly, a recent study found that pathology could be attenuated within the first 2 weeks following spinal cord injury in mice treated with an $\mathrm{N}$ cadherin neutralizing antibody, which blocked an integrin and Ncadherin dependent interaction between extracellular type-1 collagen and astrocytes and significantly attenuated astrocytic scar formation ${ }^{54}$. In this study the rapid behavioural recovery observed supports a neuroprotective role for neutralising ncadherin, though this was not assessed directly. As above, these studies suggest that early fibrosis is an important therapeutic target for improving outcome after spinal cord injury. Furthermore, if A1/A2 polarisation factors ${ }^{94}$ are conserved following spinal cord injury, a known inhibitor of $\mathrm{Clq}$ is chondroitin sulfate $\mathrm{A}^{156}$, the mono CS-4 sulfated GAG, which raises interesting questions regarding additional roles of ECM proteoglycans and their sulfation epitopes following injury. Thus, matrix properties are a valuable means to tap into the plasticity of cell responses.
CSPGs (see Box 2) are known inhibitors of neuronal plasticity, present throughout the CNS ECM and highly concentrated in the spinal injury scar (discussed above). A number of experimental studies have reported functional improvement following spinal cord injury by genetic removal of enzymes critical for CS-GAG biosynthesis. This includes deoxyribozyme-mediated knockdown of xylosyltransferase-1 mRNA, the enzyme which catalyses GAG addition to the CSPG core protein ${ }^{157}$, conditional sox9 ablation $^{158}$ and knockout of $\mathrm{N}$-acetylegalactosaminyltransferase1 , the enzyme which catalyses the addition of the first GalNAc residue onto the tetrasaccharide link between the core PG and $\mathrm{GAG}^{159}$. Reports of therapeutically-applicable pharmacological approaches which recapitulate these effects are currently lacking.

Enzymatic strategies targeting CSPGs are a promising approach for spinal cord repair, due to their ability to render the ECM more permissive to neuronal plasticity and connectivity. Removal of CS-GAGs by the chondroitinase ABC (ChABC) enzyme has been widely demonstrated to have beneficial effects in enhancing axonal regeneration and neuroplasticity and promoting functional recovery following experimental spinal cord injury ${ }^{110,160-163}$. This effect has been replicated across multiple laboratories and in different species ${ }^{164}$, including mouse, rat, cat, and recently in primates ${ }^{165}$ and in a canine clinical model ${ }^{166}$. Furthermore, its use as an adjunct therapy can augment the benefits of other experimental therapeutics ${ }^{167-169}$. A gene therapy method of enzyme delivery, where host cells are themselves transduced to express the ChABC gene leads to extensive CSGAG digestion, which results in reduced pathology and improved functional recovery following contusion injury to the thoracic ${ }^{170}$ and cervical ${ }^{171,172}$ spinal cord. Furthermore, widespread CSPG modulation achieved by viral delivery of ChABC promotes conversion of macrophages towards a pro-resolving M2 polarization state ${ }^{170}$ and drives an anti-inflammatory IL-10-mediated response $^{173}$, which is likely to underlie reduced pathology. Thus, $\mathrm{ChABC}$ is a promising means to promote resolution of pathology as well as overcoming the inhibitory environment of the spinal injury scar. A recent study utilised a novel gene switch to enable controlled delivery of the ChABC gene and revealed that long term $\mathrm{ChABC}$ gene expression was required to elicit recovery of skilled reach and grasp functions, with recovery attributed to plasticity of descending systems ${ }^{172}$. Whether this viral ChABC approach will also have benefit when applied chronically is not yet established. However, recent work has demonstrated that a single injection of ChABC enzyme in the phrenic motor pool 1.5 years after unilateral cervical spinal cord injury was able to elicit rapid and robust recovery of respiratory function, restoring the ventilatory response to the paralysed hemidiaphragm ${ }^{174}$. Furthermore, chronic application of ChABC prior to transplantation of induced pluripotent stem cell-derived neural stem cells 7 weeks after a spinal compression injury led to reduced chronic-injury scarring, increased graft survival and improved limb function ${ }^{175}$. These studies highlight the potential for ChABC to unmask latent neuroplasticity and produce a microenvironment conducive to repair even within the chronic spinal injury scar.

Another enzymatic strategy that has recently been exploited for reducing CSPG inhibition is the mammalian enzyme Arylsulfatase B (ARSB, N-acetylgalatosamine-4-sulfatase), which removes C4S moieties specifically from CS-GAGs. In addition to being utilized in enzyme-replacement therapy for human mucopolysaccharidosis VI, ARSB administration has now been shown in one study to promote increased axonal sprouting and functional locomotor recovery following compression spinal cord injury in the mouse ${ }^{176}$. ARSB perhaps represents a more attractive, and more readily translatable, therapeutic prospect than a bacterial enzyme such as ChABC and certainly warrants further 
investigation, particularly given recent findings of eliciting enhanced axon growth in the injured optic nerve ${ }^{177}$. However, whether ARSB could elicit robust modulatory effects within the spinal injury scar microenvironment, comparable to ChABC, remains to be determined. Given the specificity of ARSB for $4 S$ motifs, it may not be capable of the multi-modulatory effects that have been demonstrated for ChABC which include immune modulation, neuroprotection and neuroplasticity $164,178,179$.

Targeted modulation of CSPG receptor signalling via manipulation of the receptor PTP $\sigma$, has also proved to be a promising therapeutic prospect. The activity of the intracellular phosphatase domains of PTP $\sigma$ are regulated via a conserved "wedge" structure which can occlude the catalytic domain, thus reducing phosphorylation activity and ability to signal downstream. Use of a membrane-permeable peptide mimetic of this wedge reduces PTP $\sigma$ signalling following activation by ligands such as CSPGs. Systemic delivery of this peptide has been shown to enable recovery of locomotor and bladder function in rats following spinal contusion injury ${ }^{180}$ and the non-invasive nature of this approach means it is a potential candidate for rapid translation. Thus, approaches to overcome the inhibitory actions of CSPGs show collective promise in enabling beneficial alterations to the ECM associated with the spinal injury scar with positive effects in eliciting some functional repair.

Future directions for therapy. The majority of approaches aimed at manipulating the spinal injury scar for therapeutic benefit have focused on modifying scar-associated ECM and targeting the synthesis, production and signalling of CSPGs. With the identification of cell subtypes that have opposing actions on tissue pathology, such as A1 neurotoxic vs A2 reparatory astrocytes ${ }^{94}$, there may be further opportunity to modulate astrocyte function or phenotype following spinal cord injury. These approaches will likely evolve as new markers are identified for delineating reactive astrocytes and microglia in different phenotypic states ${ }^{181}$, with increasing availability of astrocyte and microglia cell-specific sequencing data ${ }^{54,79,108}$ and with powerful emerging tissue sequencing technology ${ }^{182}$. Alternative approaches which may indirectly modulate astrocyte phenotype are also emerging, such as grafting specific stem cell populations which can influence host tissue cellular responses and drive astrocyte transformation to a permissive phenotype ${ }^{132}$. Additionally, with increased appreciation for the role of ECM molecules in affecting pathology and plasticity of cellular responses, novel targets may be identified with new matrix biology technology ${ }^{183}$. Conversely, the study of scarring mechanisms and ECM components in organisms that are capable of CNS regeneration may lead to the identification of pro-repair targets. For example, differential regulation of collagen XII within the scar matrix is one factor contributing to the pro-regenerative phenotype in zebrafish, controlled by $\mathrm{Wnt} / \mathrm{B}$ catenin signalling 184 . Whether Wnt/B-catenin-mediated collagen XII production can be harnessed to render the mammalian spinal injury scar more permissive is not yet known. Finally, consideration should be given to scar biomechanics $^{121,125}$ when designing therapies. It may be important to understand how pharmacological manipulations affect mechanobiology and further provide appropriate mechanical signals to optimize repair.

\section{Conclusion}

The spinal injury scar is multifaceted. It contains more than just a reactive glial component and should be considered as a whole, since there is a complex interplay between multiple different cell types (glial cells, mesenchymal-derived cells, immune cells), their intracellular and signalling changes, and the extracellular environment. These processes modulate and feedback on each other. Altering the environment, for example using CSPG modulation with chondroitinase, can increase neuronal regenerationassociated gene expression and transcriptional changes ${ }^{110,173}$ Conversely, altering intracellular mechanisms can alter the inhibitory environment (for example microtubule stabilization with taxol or epothilone B leads to reduced fibrotic scarring ${ }^{148}$ ). Similarly, astrocyte-immune cell interactions are bidirectional, where an increasingly proinflammatory environment induces extensive astrogliosis ${ }^{53}$ and in turn, activated astrocytes release pro-inflammatory cytokines, chemokines and CSPGs, which can influence the magnitude of the inflammatory response ${ }^{48}$. The spinal injury scar has both beneficial properties (being essential for preventing spread of cellular damage) and detrimental properties (limiting new growth and tissue repair). This may be attributable to opposing phenotypes of reactive glial cells that form the scar border, given recent evidence in other CNS pathologies ${ }^{94}$. However, also important to note are the opposing roles of the scar matrix which contains beneficial molecules, required for formation of the glia limitans (which if not formed properly, can increase damage and worsen outcome) as well as molecules that are potent inhibitors of growth and neuroplasticity, such as CSPGs. Therapeutic strategies need to target detrimental aspects while preserving the beneficial properties of the spinal injury scar. Increased mechanistic understanding of the biological processes that propagate the non-resolving scar pathology is providing new therapeutic targets which may bring us closer to improving functional outcome following traumatic spinal cord injury.

Received: 8 November 2017 Accepted: 25 July 2019

Published online: 28 August 2019

\section{References}

1. Collaborators, G. T. B. a. S. C. I. Global, regional, and national burden of traumatic brain injury and spinal cord injury, 1990-2016: a systematic analysis for the Global Burden of Disease Study 2016. Lancet Neurol. 18, 56-87 (2019).

2. Ahuja, C. S. et al. Traumatic spinal cord injury. Nat. Rev. Dis. Prim. 3, 17018 (2017).

3. Ramer, L. M., Ramer, M. S. \& Bradbury, E. J. Restoring function after spinal cord injury: towards clinical translation of experimental strategies. Lancet Neurol. 13, 1241-1256 (2014).

4. Sofroniew, M. V. Dissecting spinal cord regeneration. Nature 557, 343-350 (2018).

5. Bradke, F., Fawcett, J. W. \& Spira, M. E. Assembly of a new growth cone after axotomy: the precursor to axon regeneration. Nat. Rev. Neurosci. 13, 183-193 (2012).

6. Puttagunta, R. et al. PCAF-dependent epigenetic changes promote axonal regeneration in the central nervous system. Nat. Commun. 5, 3527 (2014).

7. Hilton, B. J. \& Bradke, F. Can injured adult CNS axons regenerate by recapitulating development? Development. 144, 3417-3429 (2017).

8. Venkatesh, I., Mehra, V., Wang, Z., Califf, B. \& Blackmore, M. G. Developmental chromatin restriction of pro-growth gene networks acts as an epigenetic barrier to axon regeneration in cortical neurons. Dev. Neurobiol., https://doi.org/10.1002/dneu.22605 (2018).

9. Mahar, M. \& Cavalli, V. Intrinsic mechanisms of neuronal axon regeneration Nat. Rev. Neurosci. 19, 323-337 (2018).

10. Cheah, M. et al. Expression of an activated integrin promotes long-distance sensory axon regeneration in the spinal cord. J. Neurosci. 36, 7283-7297 (2016).

11. Hellal, F. et al. Microtubule stabilization reduces scarring and causes axon regeneration after spinal cord injury. Science 331, 928-931 (2011).

12. Koseki, H. et al. Selective rab11 transport and the intrinsic regenerative ability of CNS axons. eLife 6, https://doi.org/10.7554/eLife.26956 (2017).

13. Wells, J. M. \& Watt, F. M. Diverse mechanisms for endogenous regeneration and repair in mammalian organs. Nature 557, 322-328 (2018). 
14. Burda, J. E., Bernstein, A. M. \& Sofroniew, M. V. Astrocyte roles in traumatic brain injury. Exp. Neurol. 275, 305-315 (2016).

15. Sims, N. R. \& Yew, W. P. Reactive astrogliosis in stroke: contributions of astrocytes to recovery of neurological function. Neurochem. Int. 107, 88-103 (2017).

16. Rodriguez, J. J., Butt, A. M., Gardenal, E., Parpura, V. \& Verkhratsky, A. Complex and differential glial responses in Alzheimer's disease and ageing. Curr. Alzheimer Res. 13, 343-358 (2016).

17. Ponath, G., Park, C. \& Pitt, D. The Role of Astrocytes in multiple sclerosis. Front. Immunol. 9, 217 (2018).

18. Stephenson, E. L. et al. Chondroitin sulfate proteoglycans as novel drivers of leucocyte infiltration in multiple sclerosis. Brain 141, 1094-1110 (2018).

19. Adams, K. L. \& Gallo, V. The diversity and disparity of the glial scar. Nat. Neurosci. 21, 9-15 (2018).

20. Schnell, L., Fearn, S., Klassen, H., Schwab, M. E. \& Perry, V. H. Acute inflammatory responses to mechanical lesions in the CNS: differences between brain and spinal cord. Eur. J. Neurosci. 11, 3648-3658 (1999).

21. Anthony, D. C. \& Couch Y. The systemic response to CNS injury. Exp. Neurol. 258, 105-111 (2014)

22. Zhang, B. \& Gensel, J. C. Is neuroinflammation in the injured spinal cord different than in the brain? Examining intrinsic differences between the brain and spinal cord. Exp. Neurol. 258, 112-120 (2014).

23. Rowlands, D., Sugahara, K. \& Kwok, J. C. Glycosaminoglycans and glycomimetics in the central nervous system. Molecules 20, 3527-3548 (2015).

24. Smith, P. D., Coulson-Thomas, V. J., Foscarin, S., Kwok, J. C. \& Fawcett, J. W. "GAG-ing with the neuron": The role of glycosaminoglycan patterning in the central nervous system. Exp. Neurol. 274, 100-114 (2015).

25. Miller, G. M. \& Hsieh-Wilson, L. C. Sugar-dependent modulation of neuronal development, regeneration, and plasticity by chondroitin sulfate proteoglycans. Exp. Neurol., https://doi.org/10.1016/j.expneurol.2015.08.015 (2015).

26. Cregg, J. M. et al. Functional regeneration beyond the glial scar. Exp. Neurol. 253, 197-207 (2014).

27. Fawcett, J. W., Schwab, M. E., Montani, L., Brazda, N. \& Muller, H. W. Defeating inhibition of regeneration by scar and myelin components. Handb. Clin. Neurol. 109, 503-522 (2012).

28. Busch, S. A. et al. Adult NG2+ cells are permissive to neurite outgrowth and stabilize sensory axons during macrophage-induced axonal dieback after spinal cord injury. J. Neurosci. 30, 255-265 (2010).

29. Ramón y Cajal, S. Degeneration and Regeneration in the Nervous System (Oxford University Press, Oxford, 1928).

30. Reier, P. J., Stensaas, L. J. \& Guth, L. The astrocytic scar as an impediment to regeneration in the central nervous system. In Spinal Cord Reconstruction (ed Bunge, R. P., Kao, C. C. \& Reier, P. J.) 163-195 (Raven Press, New York, 1983).

31. O'Shea, T. M., Burda, J. E. \& Sofroniew, M. V. Cell biology of spinal cord injury and repair. J. Clin. Investig. 127, 3259-3270 (2017).

32. Silver, J. \& Miller, J. H. Regeneration beyond the glial scar. Nat. Rev. Neurosci. 5, 146-156 (2004).

33. Klapka, N. \& Muller, H. W. Collagen matrix in spinal cord injury. J. neurotrauma 23, 422-435 (2006).

34. Mautes, A. E., Weinzierl, M. R., Donovan, F. \& Noble, L. J. Vascular events after spinal cord injury: contribution to secondary pathogenesis. Phys. Ther. 80, 673-687 (2000).

35. Davalos, D. et al. ATP mediates rapid microglial response to local brain injury in vivo. Nat. Neurosci. 8, 752-758 (2005).

36. Haynes, S. E. et al. The $\mathrm{P} 2 \mathrm{Y} 12$ receptor regulates microglial activation by extracellular nucleotides. Nat. Neurosci. 9, 1512-1519 (2006)

37. Wu, L. J., Vadakkan, K. I. \& Zhuo, M. ATP-induced chemotaxis of microglial processes requires $\mathrm{P} 2 \mathrm{Y}$ receptor-activated initiation of outward potassium currents. Glia 55, 810-821 (2007).

38. Bellver-Landete, V. et al. Microglia are an essential component of the neuroprotective scar that forms after spinal cord injury. Nat. Commun. 10, 518 (2019)

39. Huang, C. et al. Critical role of connexin 43 in secondary expansion of traumatic spinal cord injury. J. Neurosci. 32, 3333-3338 (2012)

40. James, G. \& Butt, A. M. P2Y and P2X purinoceptor mediated Ca2+ signalling in glial cell pathology in the central nervous system. Eur. J. Pharm. 447, 247-260 (2002).

41. Franke, H. et al. P2 receptor-types involved in astrogliosis in vivo. Br. J. Pharmacol. 134, 1180-1189 (2001).

42. Park, E., Velumian, A. A. \& Fehlings, M. G. The role of excitotoxicity in secondary mechanisms of spinal cord injury: a review with an emphasis on the implications for white matter degeneration. J. Neurotrauma 21, 754-774 (2004).

43. David, S., Greenhalgh, A. D. \& Lopez-Vales, R. Role of phospholipase A2s and lipid mediators in secondary damage after spinal cord injury. Cell tissue Res. 349, 249-267 (2012).
44. Simard, J. M. et al. Endothelial sulfonylurea receptor 1-regulated NC Ca-ATP channels mediate progressive hemorrhagic necrosis following spinal cord injury. J. Clin. Investig. 117, 2105-2113 (2007).

45. Goodman, J. H., Bingham, W. G. Jr. \& Hunt, W. E. Platelet aggregation in experimental spinal cord injury. Ultrastructural observations. Arch. Neurol. 36, 197-201 (1979)

46. Matzinger, P. Tolerance danger, and the extended family. Аnnu. Rev. Immunol. 12, 991-1045 (1994).

47. Chen, G. Y. \& Nunez, G. Sterile inflammation: sensing and reacting to damage. Nat. Rev. Immunol. 10, 826-837 (2010).

48. Gaudet, A. D. \& Popovich, P. G. Extracellular matrix regulation of inflammation in the healthy and injured spinal cord. Exp. Neurol. 0, 24-34 (2014).

49. Pineau, I. \& Lacroix, S. Proinflammatory cytokine synthesis in the injured mouse spinal cord: multiphasic expression pattern and identification of the cell types involved. J. Comp. Neurol. 500, 267-285 (2007).

50. Didangelos, A. et al. High-throughput proteomics reveal alarmins as amplifiers of tissue pathology and inflammation after spinal cord injury. Sci. Rep. 6, 21607 (2016).

51. Sofroniew, M. V. Multiple roles for astrocytes as effectors of cytokines and inflammatory mediators. Neurosci.: a Rev. J. Bringing Neurobiol., Neurol. Psychiatry 20, 160-172 (2014)

52. Anderson, M. A., Ao, Y. \& Sofroniew, M. V. Heterogeneity of reactive astrocytes. Neurosci. Lett. 565, 23-29 (2014).

53. Sofroniew, M. V. Astrogliosis. Cold Spring Harb. Perspect. Biol. 7, a020420 (2014).

54. Hara, M. et al. Interaction of reactive astrocytes with type I collagen induces astrocytic scar formation through the integrin-N-cadherin pathway after spinal cord injury. Nat. Med. https://doi.org/10.1038/nm.4354 (2017).

55. Zamanian, J. L. et al. Genomic analysis of reactive astrogliosis. J. Neurosci. 32, 6391-6410 (2012).

56. Chen, $M$. et al. Leucine zipper-bearing kinase is a critical regulator of astrocyte reactivity in the adult mammalian CNS. Cell Rep. 22, 3587-3597 (2018).

57. Sun, D. \& Jakobs, T. C. Structural remodeling of astrocytes in the injured CNS. Neurosci.: a Rev. J. Bringing Neurobiol., Neurol. Psychiatry 18, 567-588 (2012).

58. Herrmann, J. E. et al. STAT3 is a critical regulator of astrogliosis and scar formation after spinal cord injury. J. Neurosci. 28, 7231-7243 (2008).

59. Wanner, I. B. et al. Glial scar borders are formed by newly proliferated, elongated astrocytes that interact to corral inflammatory and fibrotic cells via STAT3-dependent mechanisms after spinal cord injury. J. Neurosci. 33, 12870-12886 (2013)

60. Sabelstrom, $\mathrm{H}$. et al. Resident neural stem cells restrict tissue damage and neuronal loss after spinal cord injury in mice. Science 342, 637-640 (2013).

61. Ren, Y. et al. Ependymal cell contribution to scar formation after spinal cord injury is minimal, local and dependent on direct ependymal injury. Sci. Rep. 7, 41122 (2017).

62. Bundesen, L. Q., Scheel, T. A., Bregman, B. S. \& Kromer, L. F. Ephrin-B2 and EphB2 regulation of astrocyte-meningeal fibroblast interactions in response to spinal cord lesions in adult rats. J. Neurosci. 23, 7789-7800 (2003).

63. Soderblom, C. et al. Perivascular fibroblasts form the fibrotic scar after contusive spinal cord injury. J. Neurosci. 33, 13882-13887 (2013).

64. Sofroniew, M. V. Molecular dissection of reactive astrogliosis and glial scar formation. Trends Neurosci. 32, 638-647 (2009).

65. Okada, M. et al. Expression and role of type I collagen in a rat spinal cord contusion injury model. Neurosci. Res. 58, 371-377 (2007).

66. Göritz, C. et al. A Pericyte Origin of Spinal Cord Scar Tissue. Science 333 238-242 (2011)

67. Dias, D. O. et al. Reducing Pericyte-Derived Scarring Promotes Recovery after Spinal Cord Injury. Cell 173, 153-165.e122 (2018).

68. Birbrair, A. et al. Type-1 pericytes accumulate after tissue injury and produce collagen in an organ-dependent manner. Stem Cell Res. Ther. 5, 122 (2014).

69. McTigue, D. M., Tripathi, R. \& Wei, P. NG2 colocalizes with axons and is expressed by a mixed cell population in spinal cord lesions. J. Neuropathol. Exp. Neurol. 65, 406-420 (2006).

70. Yokota, K. et al. Periostin promotes scar formation through the interaction between pericytes and infiltrating monocytes/macrophages after spinal cord injury. Am. J. Pathol. 187, 639-653 (2017).

71. Hackett, A. R. \& Lee, J. K. Understanding the NG2 glial scar after spinal cord injury. Front. Neurol. 7, 199 (2016).

72. Levine, J. The reactions and role of NG2 glia in spinal cord injury. Brain Res 1638, 199-208 (2016).

73. Assinck, P. et al. Myelinogenic plasticity of oligodendrocyte precursor cells following spinal cord contusion injury. J. Neurosci. 37, 8635-8654 (2017).

74. Bartus, K. et al. ErbB receptor signaling directly controls oligodendrocyte progenitor cell transformation and spontaneous remyelination after spinal cord injury. Glia, https://doi.org/10.1002/glia.23586 (2019). 
75. Hesp, Z. C., Goldstein, E. Z., Miranda, C. J., Kaspar, B. K. \& McTigue, D. M. Chronic oligodendrogenesis and remyelination after spinal cord injury in mice and rats. J. Neurosci. 35, 1274-1290 (2015).

76. Filous, A. R. et al. Entrapment via synaptic-like connections between NG2 proteoglycan + cells and dystrophic axons in the lesion plays a role in regeneration failure after spinal cord injury. J. Neurosci. 34, 16369-16384 (2014).

77. Hackett, A. R. et al. STAT3 and SOCS3 regulate NG2 cell proliferation and differentiation after contusive spinal cord injury. Neurobiol. Dis. 89, 10-22 (2016).

78. Duncan, G. J. et al. Locomotor recovery following contusive spinal cord injury does not require oligodendrocyte remyelination. Nat. Commun. 9, 3066 (2018).

79. Bennett, M. L. et al. New tools for studying microglia in the mouse and human CNS. Proc. Natl Acad. Sci. USA 113, E1738-E1746 (2016).

80. David, S., Kroner, A., Greenhalgh, A. D., Zarruk, J. G. \& López-Vales, R. Myeloid cell responses after spinal cord injury. J. Neuroimmunol. 321, 97-108 (2018).

81. Kaiser, T. \& Feng, G. Tmem119-EGFP and Tmem119-CreERT2 transgenic mice for labeling and manipulating microglia. bioRxiv, 624825, https://doi.org/ 10.1101/624825 (2019).

82. Ruan, C. et al. A novel Tmem119-tdTomato REporter mouse model for studying microglia in the central nervous system. bioRxiv, 665893, https://doi. org/10.1101/665893 (2019)

83. Kigerl, K. A. et al. Identification of two distinct macrophage subsets with divergent effects causing either neurotoxicity or regeneration in the injured mouse spinal cord. J. Neurosci. 29, 13435-13444 (2009).

84. Gensel, J. C. \& Zhang, B. Macrophage activation and its role in repair and pathology after spinal cord injury. Brain Res. 1619, 1-11 (2015).

85. Greenhalgh, A. D. \& David, S. Differences in the phagocytic response of microglia and peripheral macrophages after spinal cord injury and its effects on cell death. J. Neurosci. 34, 6316-6322 (2014).

86. Sun, G. et al. gammadelta T cells provide the early source of IFN-gamma to aggravate lesions in spinal cord injury. J. Exp. Med. 215, 521-535 (2018).

87. Popovich, P. G., Stokes, B. T. \& Whitacre, C. C. Concept of autoimmunity following spinal cord injury: possible roles for T lymphocytes in the traumatized central nervous system. J. Neurosci. Res. 45, 349-363 (1996).

88. Ankeny, D. P., Guan, Z. \& Popovich, P. G. B cells produce pathogenic antibodies and impair recovery after spinal cord injury in mice. J. Clin. Investig. 119, 2990-2999 (2009).

89. Donnelly, D. J. \& Popovich, P. G. Inflammation and its role in neuroprotection, axonal regeneration and functional recovery after spinal cord injury. Exp. Neurol. 209, 378-388 (2008).

90. Pruss, $H$. et al. Non-resolving aspects of acute inflammation after spinal cord injury (SCI): indices and resolution plateau. Brain Pathol. 21, 652-660 (2011).

91. Vannella, K. M. \& Wynn, T. A. Mechanisms of organ injury and repair by macrophages. Annu. Rev. Physiol. 79, 593-617 (2017).

92. Okada, S. et al. Conditional ablation of Stat 3 or Socs 3 discloses a dual role for reactive astrocytes after spinal cord injury. Nat. Med. 12, 829-834 (2006).

93. Brambilla, R. et al. Inhibition of astroglial nuclear factor kappaB reduces inflammation and improves functional recovery after spinal cord injury. $J$. Exp. Med. 202, 145-156 (2005).

94. Liddelow, S. A. et al. Neurotoxic reactive astrocytes are induced by activated microglia. Nature 541, 481-487 (2017).

95. Rodriguez, J. P. et al. Abrogation of beta-catenin signaling in oligodendrocyte precursor cells reduces glial scarring and promotes axon regeneration after CNS injury. J. Neurosci. 34, 10285-10297 (2014).

96. Horn, K. P., Busch, S. A., Hawthorne, A. L., van Rooijen, N. \& Silver, J. Another barrier to regeneration in the CNS: activated macrophages induce extensive retraction of dystrophic axons through direct physical interactions. J. Neurosci. 28, 9330-9341 (2008).

97. Evans, T. A. et al. High-resolution intravital imaging reveals that bloodderived macrophages but not resident microglia facilitate secondary axonal dieback in traumatic spinal cord injury. Exp. Neurol. 254, 109-120 (2014).

98. Andrews, E. M., Richards, R. J., Yin, F. Q., Viapiano, M. S. \& Jakeman, L. B. Alterations in chondroitin sulfate proteoglycan expression occur both at and far from the site of spinal contusion injury. Exp. Neurol. 235, 174-187 (2012).

99. Steward, O. \& Trimmer, P. A. Genetic influences on cellular reactions to CNS injury: the reactive response of astrocytes in denervated neuropil regions in mice carrying a mutation (Wld(S)) that causes delayed Wallerian degeneration. J. Comp. Neurol. 380, 70-81 (1997).

100. Buss, A. et al. Gradual loss of myelin and formation of an astrocytic scar during Wallerian degeneration in the human spinal cord. Brain 127, 34-44 (2004).

101. Pizzorusso, T. et al. Reactivation of ocular dominance plasticity in the adult visual cortex. Science 298, 1248-1251 (2002).
102. Zhu, Y. et al. Macrophage transcriptional profile identifies lipid catabolic pathways that can be therapeutically targeted after spinal cord injury. J. Neurosci. 37, 2362-2376 (2017).

103. Zhu, Y. et al. Hematogenous macrophage depletion reduces the fibrotic scar and increases axonal growth after spinal cord injury. Neurobiol. Dis. 74, 114-125 (2015).

104. Schwab, M. E. \& Strittmatter, S. M. Nogo limits neural plasticity and recovery from injury. Curr. Opin. Neurobiol. 27, 53-60 (2014).

105. Niederost, B., Oertle, T., Fritsche, J., McKinney, R. A. \& Bandtlow, C. E. Nogo$\mathrm{A}$ and myelin-associated glycoprotein mediate neurite growth inhibition by antagonistic regulation of RhoA and Racl. J. Neurosci. 22, 10368-10376 (2002).

106. McKeon, R. J., Jurynec, M. J. \& Buck, C. R. The chondroitin sulfate proteoglycans neurocan and phosphacan are expressed by reactive astrocytes in the chronic CNS glial scar. J. Neurosci. 19, 10778-10788 (1999).

107. Davies, S. J., Goucher, D. R., Doller, C. \& Silver, J. Robust regeneration of adult sensory axons in degenerating white matter of the adult rat spinal cord. $J$. Neurosci. 19, 5810-5822 (1999).

108. Anderson, M. A. et al. Astrocyte scar formation aids central nervous system axon regeneration. Nature 532, 195-200 (2016)

109. Ughrin, Y. M., Chen, Z. J. \& Levine, J. M. Multiple regions of the NG2 proteoglycan inhibit neurite growth and induce growth cone collapse. J. Neurosci. 23, 175-186 (2003).

110. Bradbury, E. J. et al. Chondroitinase $\mathrm{ABC}$ promotes functional recovery after spinal cord injury. Nature 416, 636-640 (2002)

111. Shen, Y. et al. PTPsigma is a receptor for chondroitin sulfate proteoglycan, an inhibitor of neural regeneration. Science 326, 592-596 (2009).

112. Fry, E. J., Chagnon, M. J., Lopez-Vales, R., Tremblay, M. L. \& David, S Corticospinal tract regeneration after spinal cord injury in receptor protein tyrosine phosphatase sigma deficient mice. Glia 58, 423-433 (2010).

113. Fisher, D. et al. Leukocyte common antigen-related phosphatase is a functional receptor for chondroitin sulfate proteoglycan axon growth inhibitors. J. Neurosci. 31, 14051-14066 (2011).

114. Dickendesher, T. L. et al. NgR1 and NgR3 are receptors for chondroitin sulfate proteoglycans. Nat. Neurosci. 15, 703-712 (2012).

115. Sivasankaran, R. et al. PKC mediates inhibitory effects of myelin and chondroitin sulfate proteoglycans on axonal regeneration. Nat. Neurosci. 7, 261-268 (2004)

116. Koprivica, V. et al. EGFR activation mediates inhibition of axon regeneration by myelin and chondroitin sulfate proteoglycans. Science 310, 106-110 (2005)

117. Dill, J., Wang, H., Zhou, F. \& Li, S. Inactivation of glycogen synthase kinase 3 promotes axonal growth and recovery in the CNS. J. Neurosci. 28, 8914-8928 (2008).

118. Tan, C. L. et al. Integrin activation promotes axon growth on inhibitory chondroitin sulfate proteoglycans by enhancing integrin signaling. J. Neurosci. 31, 6289-6295 (2011).

119. De Winter, F. et al. Injury-induced class 3 semaphorin expression in the rat spinal cord. Exp. Neurol. 175, 61-75 (2002).

120. Bradbury, E. J. \& Burnside, E. R. Strategies to overcome the inhibitory environment of the spinal cord. In AOSpine Masters Series (ed Luiz Roberto Vialle) (Georg Thieme Verlag, Stuttgart, 2017).

121. Franze, K., Janmey, P. A. \& Guck, J. Mechanics in neuronal development and repair. Annu Rev. Biomed. Eng. 15, 227-251 (2013)

122. Lo, C. M., Wang, H. B., Dembo, M. \& Wang, Y. L. Cell movement is guided by the rigidity of the substrate. Biophys. J. 79, 144-152 (2000).

123. Koser, D. E. et al. Mechanosensing is critical for axon growth in the developing brain. Nat. Neurosci. 19, 1592-1598 (2016).

124. Moshayedi, P. et al. Mechanosensitivity of astrocytes on optimized polyacrylamide gels analyzed by quantitative morphometry. Journal of physics. Condens. Matter. 22, 194114 (2010).

125. Moeendarbary, E. et al. The soft mechanical signature of glial scars in the central nervous system. Nat. Commun. 8, 14787 (2017).

126. Grant, C. A., Twigg, P. C. \& Tobin, D. J. Static and dynamic nanomechanical properties of human skin tissue using atomic force microscopy: effect of scarring in the upper dermis. Acta Biomater. 8, 4123-4129 (2012).

127. Swift, J. et al. Nuclear lamin-A scales with tissue stiffness and enhances matrix-directed differentiation. Science 341, 1240104 (2013).

128. Weickenmeier, J. et al. Brain stiffness increases with myelin content. Acta Biomater. 42, 265-272 (2016).

129. Zhou, T. et al. Microvascular endothelial cells engulf myelin debris and promote macrophage recruitment and fibrosis after neural injury. Nat. Neurosci. 22, 421-435 (2019).

130. Liddelow, S. A. \& Barres, B. A. Regeneration: not everything is scary about a glial scar. Nature 532, 182-183 (2016).

131. Silver, J. The glial scar is more than just astrocytes. Exp. Neurol. https://doi. org/10.1016/j.expneurol.2016.06.018 (2016).

132. Lukovic, D. et al. Concise review: reactive astrocytes and stem cells in spinal cord injury: good guys or bad guys? Stem Cells 33, 1036-1041 (2015) 
133. Davies, S. J. et al. Regeneration of adult axons in white matter tracts of the central nervous system. Nature 390, 680-683 (1997).

134. Sandvig, A., Berry, M., Barrett, L. B., Butt, A. \& Logan, A. Myelin-, reactive glia-, and scar-derived CNS axon growth inhibitors: expression, receptor signaling, and correlation with axon regeneration. Glia 46, 225-251 (2004).

135. Das, G. D. in Neural Transplantation and Regeneration (ed Gopal D. Das, Wallace \& Robert. B) (Springer Verlag, Berlin, Germany, 1986).

136. Mathewson, A. J. \& Berry, M. Observations on the astrocyte response to a cerebral stab wound in adult rats. Brain Res. 327, 61-69 (1985).

137. Kawano, H., Kimura-Kuroda, J., Komuta, Y., Yoshioka, N., Li, H. P., Kawamura, K., Li, Y. \& Raisman, G. Role of the lesion scar in the response to damage and repair of the central nervous system. Cell Tissue Res. 349, 169-180 (2012).

138. Pekny, M. et al. Abnormal reaction to central nervous system injury in mice lacking glial fibrillary acidic protein and vimentin. J. Cell Biol. 145, 503-514 (1999).

139. Bush, T. G. et al. Leukocyte infiltration, neuronal degeneration, and neurite outgrowth after ablation of scar-forming, reactive astrocytes in adult transgenic mice. Neuron 23, 297-308 (1999).

140. Faulkner, J. R. et al. Reactive astrocytes protect tissue and preserve function after spinal cord injury. J. Neurosci. 24, 2143-2155 (2004).

141. Tran, A. P., Warren, P. M. \& Silver, J. The Biology of Regeneration Failure and Success After Spinal Cord Injury. Physiol. Rev. 98, 881-917 (2018).

142. Rolls, A., Shechter, R. \& Schwartz, M. The bright side of the glial scar in CNS repair. Nat. Rev. Neurosci. 10, 235-241 (2009).

143. Karimi-Abdolrezaee, S. \& Billakanti, R. Reactive astrogliosis after spinal cord injury-beneficial and detrimental effects. Mol. Neurobiol. 46, 251-264 (2012).

144. Li, X. et al. Comparison of subacute and chronic scar tissues after complete spinal cord transection. Exp. Neurol. 306, 132-137 (2018).

145. White, R. E. \& Jakeman, L. B. Don't fence me in: harnessing the beneficial roles of astrocytes for spinal cord repair. Restor. Neurol. Neurosci. 26, 197-214 (2008).

146. Smith, J. S. et al. Role of early surgical decompression of the intradural space after cervical spinal cord injury in an animal model. J. Bone Jt. Surg. Am. Vol. 92, 1206-1214 (2010).

147. Phang, I. et al. Expansion duroplasty improves intraspinal pressure, spinal cord perfusion pressure, and vascular pressure reactivity index in patients with traumatic spinal cord injury: injured spinal cord pressure evaluation study. $J$. Neurotrauma 32, 865-874 (2015).

148. Ruschel, J. et al. Axonal regeneration. Systemic administration of epothilone B promotes axon regeneration after spinal cord injury. Science 348, 347-352 (2015).

149. Forli, S. Epothilones: from discovery to clinical trials. Curr. Top. Med. Chem. 14, 2312-2321 (2014).

150. Klapka, N. et al. Suppression of fibrous scarring in spinal cord injury of rat promotes long-distance regeneration of corticospinal tract axons, rescue of primary motoneurons in somatosensory cortex and significant functional recovery. Eur. J. Neurosci. 22, 3047-3058 (2005).

151. Brazda, N. \& Muller, H. W. Pharmacological modification of the extracellular matrix to promote regeneration of the injured brain and spinal cord. Prog. Brain Res. 175, 269-281 (2009).

152. Vogelaar, C. F. et al. Pharmacological suppression of CNS scarring by deferoxamine reduces lesion volume and increases regeneration in an in vitro model for astroglial-fibrotic scarring and in rat spinal cord injury in vivo. PLoS ONE 10, e0134371 (2015).

153. Gilad, G. M. \& Gilad, V. H. $\beta$-Aminopropionitrile treatment can accelerate recovery of mice after spinal cord injury. Eur. J. Pharmacol. 430, 69-72 (2001).

154. Esmaeili, M., Berry, M., Logan, A. \& Ahmed, Z. Decorin treatment of spinal cord injury.Neural Regeneration Res. 9, 1653-1656 (2014).

155. Occleston, N. L. et al. Discovery and development of avotermin (recombinant human transforming growth factor beta 3): a new class of prophylactic therapeutic for the improvement of scarring. Wound Repair Regen.: Off. Publ. Wound Heal. Soc. [] Eur. Tissue Repair Soc. 19(Suppl 1), s38-s48 (2011).

156. Silvestri, L., Baker, J. R., Roden, L. \& Stroud, R. M. The C1q inhibitor in serum is a chondroitin 4-sulfate proteoglycan. J. Biol. Chem. 256, 7383-7387 (1981).

157. Grimpe, B. \& Silver, J. A novel DNA enzyme reduces glycosaminoglycan chains in the glial scar and allows microtransplanted dorsal root ganglia axons to regenerate beyond lesions in the spinal cord. J. Neurosci. 24, 1393-1397 (2004).

158. McKillop, W. M., Dragan, M., Schedl, A. \& Brown, A. Conditional Sox9 ablation reduces chondroitin sulfate proteoglycan levels and improves motor function following spinal cord injury. Glia 61, 164-177 (2013).

159. Takeuchi, K. et al. Chondroitin sulphate $\mathrm{N}$-acetylgalactosaminyl-transferase-1 inhibits recovery from neural injury. Nat Commun 4, https://doi.org/10.1038/ ncomms3740 (2013).

160. Garcia-Alias, G., Barkhuysen, S., Buckle, M., Fawcett, J. W. \& Chondroitinase, A. B. C. treatment opens a window of opportunity for task-specific rehabilitation. Nat. Neurosci. 12, 1145-1151 (2009).
161. Alilain, W. J., Horn, K. P., Hu, H., Dick, T. E. \& Silver, J. Functional regeneration of respiratory pathways after spinal cord injury. Nature 475, 196-200 (2011).

162. Cafferty, W. B., Yang, S. H., Duffy, P. J., Li, S. \& Strittmatter, S. M. Functional axonal regeneration through astrocytic scar genetically modified to digest chondroitin sulfate proteoglycans. J. Neurosci. 27, 2176-2185 (2007).

163. Bowes, C. et al. Chondroitinase ABC promotes selective reactivation reactivation of somatosensory cortex in squirrel monkeys after a cervical dorsal column lesion. Proc. Natl Acad. Sci. USA 109, 2595-2600 (2012).

164. Bradbury, E. J. \& Carter, L. M. Manipulating the glial scar: chondroitinase $\mathrm{ABC}$ as a therapy for spinal cord injury. Brain Res. Bull. 84, 306-316 (2011).

165. Rosenzweig, E. S. et al. Chondroitinase improves anatomical and functional outcomes after primate spinal cord injury. Nat. Neurosci., https://doi.org/ 10.1038/s41593-019-0424-1 (2019).

166. Hu, H. Z., Granger, N., Pai, S. B., Bellamkonda, R. V. \& Jeffery, N. D. Therapeutic efficacy of microtube-embedded chondroitinase $A B C$ in a canine clinical model of spinal cord injury. Brain 141, 1017-1027 (2018).

167. Kanno, H. et al. Combination of engineered Schwann cell grafts to secrete neurotrophin and chondroitinase promotes axonal regeneration and locomotion after spinal cord injury. J. Neurosci. 34, 1838-1855 (2014).

168. Tom, V. J. et al. Combining peripheral nerve grafts and chondroitinase promotes functional axonal regeneration in the chronically injured spinal cord. J. Neurosci.: Off. J. Soc. Neurosci. 29, 14881-14890 (2009).

169. Alluin, O. et al. Examination of the combined effects of chondroitinase ABC, growth factors and locomotor training following compressive spinal cord injury on neuroanatomical plasticity and kinematics. PLoS ONE 9, el11072 (2014).

170. Bartus, K. et al. Large-scale chondroitin sulfate proteoglycan digestion with chondroitinase gene therapy leads to reduced pathology and modulates macrophage phenotype following spinal cord contusion injury. J. Neurosci.: Off. J. Soc. Neurosci. 34, 4822-4836 (2014).

171. James, N. D. et al. Chondroitinase gene therapy improves upper limb function following cervical contusion injury. Exp. Neurol. 271, 131-135 (2015).

172. Burnside, E. R. et al. Immune-evasive gene switch enables regulated delivery of chondroitinase after spinal cord injury. Brain. https://doi.org/10.1093/brain/ awy158 (2018).

173. Didangelos, A., Iberl, M., Vinsland, E., Bartus, K. \& Bradbury, E. J. Regulation of IL-10 by chondroitinase $\mathrm{ABC}$ promotes a distinct immune response following spinal cord injury. J. Neurosci.: Off. J. Soc. Neurosci. 34, 16424-16432 (2014).

174. Warren, P. M. et al. Rapid and robust restoration of breathing long after spinal cord injury. Nat. Commun. 9, 4843 (2018).

175. Suzuki, H. et al. Neural stem cell mediated recovery is enhanced by Chondroitinase $\mathrm{ABC}$ pretreatment in chronic cervical spinal cord injury. PLoS One 12, e0182339 (2017).

176. Yoo, M. et al. Arylsulfatase B Improves Locomotor Function after Mouse Spinal Cord Injury. PLoS ONE 8, e57415 (2013).

177. Pearson, C. S., Mencio, C. P., Barber, A. C., Martin, K. R. \& Geller, H. M. Identification of a critical sulfation in chondroitin that inhibits axonal regeneration. eLife 7, https://doi.org/10.7554/eLife.37139 (2018).

178. Hryciw, T., Geremia, N. M., Walker, M. A., Xu, X. \& Brown, A. Antichondroitin sulfate proteoglycan strategies in spinal cord injury: temporal and spatial considerations explain the balance between neuroplasticity and neuroprotection. J. neurotrauma 35, 1958-1969 (2018).

179. Fawcett, J. W. The extracellular matrix in plasticity and regeneration after CNS injury and neurodegenerative disease. Prog. Brain Res. 218, 213-226 (2015).

180. Lang, B. T. et al. Modulation of the proteoglycan receptor PTPsigma promotes recovery after spinal cord injury. Nature 518, 404-408 (2015).

181. Liddelow, S. A. \& Barres, B. A. Reactive astrocytes: production, function, and therapeutic potential. Immunity 46, 957-967 (2017).

182. Wang, X. et al. Three-dimensional intact-tissue sequencing of single-cell transcriptional states. Science, https://doi.org/10.1126/science.aat5691 (2018).

183. Thompson, J. W., Sorum, A. W. \& Hsieh-Wilson, L. C. Deciphering the functions of O-GlcNAc glycosylation in the brain: The role of site-specific quantitative O-GlcNAcomics. Biochemistry, https://doi.org/10.1021/acs. biochem.8b00516 (2018).

184. Tsarouchas, T. M. et al. Dynamic control of proinflammatory cytokines Illbeta and Tnf-alpha by macrophages in zebrafish spinal cord regeneration. Nat. Commun. 9, 4670 (2018).

185. Bohlen, C. J. et al. Diverse requirements for microglial survival, specification, and function revealed by defined-medium cultures. Neuron 94, 759-773.e758 (2017).

186. Mikami, T. \& Kitagawa, H. Biosynthesis and function of chondroitin sulfate. Biochim. et. Biophys. Acta 1830, 4719-4733 (2013).

\section{Acknowledgements}

E.J.B. receives funding from the U.K. Medical Research Council (SNCF G1002055; MR/ P012418/1; ERA-NET NEURON MR/R005532/1), the International Spinal Research Trust (CHASE-IT II_02), the National Institutes of Health (2 R01 GM 093627-05) and the Rosetrees Trust (A1384). 


\section{Author contributions}

E.J.B. and E.R.B. wrote the Review; E.R.B. prepared the figures, with input from E.J.B.

\section{Additional information}

Competing interests: The authors declare no competing interests.

Reprints and permission information is available online at http://npg.nature.com/ reprintsandpermissions/

Peer review information: Nature Communications thanks John Houle, Angelo Lepore and Stefanie Robel for their contribution to the peer review of this work.

Publisher's note: Springer Nature remains neutral with regard to jurisdictional claims in published maps and institutional affiliations. (c) (i) Open Access This article is licensed under a Creative Commons Attribution 4.0 International License, which permits use, sharing, adaptation, distribution and reproduction in any medium or format, as long as you give appropriate credit to the original author(s) and the source, provide a link to the Creative Commons license, and indicate if changes were made. The images or other third party material in this article are included in the article's Creative Commons license, unless indicated otherwise in a credit line to the material. If material is not included in the article's Creative Commons license and your intended use is not permitted by statutory regulation or exceeds the permitted use, you will need to obtain permission directly from the copyright holder. To view a copy of this license, visit http://creativecommons.org/ licenses/by/4.0/.

(c) The Author(s) 2019 